\title{
Turing-Hopf Patterns on Growing Domains: the Torus and the Sphere
}

\author{
Faustino Sánchez-Garduño \\ Departamento de Matemáticas, Facultad de Ciencias, Universidad Nacional Autónoma de México \\ (UNAM). Circuito Exterior, Ciudad Universitaria, Ciudad de México, Del. Coyoacán, C.P. 04510, México. \\ faustinos403@gmail.com \\ Andrew L. Krause \\ Wolfson Centre for Mathematical Biology, Mathematical Institute, University of Oxford, Andrew Wiles \\ Building, Radcliffe Observatory Quarter, Woodstock Rd, Oxford OX2 6GG, United Kingdom \\ *Corresponding author, krause@maths.ox.ac.uk \\ Jorge A. Castillo \\ Facultad de Matemáticas, Universidad Autónoma de Guerrero, Carlos E. Adame \# 54, Col. Garita, C.P. \\ 39650, Acapulco, Guerrero, México. \\ jcastillo7701@gmail.com \\ Pablo Padilla \\ IIMAS-UNAM, Apartado Postal 20-726, Admon. No. 20. C.P. 01000, Del. Álvaro Obregón, Ciudad de \\ México, México. \\ pabpad@gmail.com
}

\begin{abstract}
This paper deals with the study of spatial and spatio-temporal patterns in the reactiondiffusion FitzHugh-Nagumo model on growing curved domains. This is carried out on two exemplar cases: a torus and a sphere. We compute bifurcation boundaries for the homogeneous steady state when the homogeneous system is monostable. We exhibit Turing and Turing-Hopf bifurcations, as well as additional patterning outside of these bifurcation regimes due to the multistability of patterned states. We consider static and growing domains, where the growth is slow, isotropic, and exponential in time, allowing for a simple analytical calculation of these bifurcations in terms of model parameters. Numerical simulations allow us to discuss the role played by the growth and the curvature of the domains on the pattern selection on the torus and the sphere. We demonstrate parameter regimes where the linear theory can successfully predict the kind of pattern (homogeneous and heterogeneous oscillations and stationary spatial patterns) but not their detailed nonlinear structure. We also find parameter regimes where the linear theory fails, such as Hopf regimes which give rise to spatial patterning (depending on geometric details), where we suspect that multistability plays a key role in the departure from homogeneity. Finally we also demonstrate effects due to the evolution of nonuniform patterns under growth, suggesting important roles for growth
\end{abstract}


in reaction-diffusion systems beyond modifying instability regimes.

Keywords: Pattern formation, Turing-Hopf bifurcation, isotropically growing domains, FitzHugh-Nagumo model.

\section{Introduction}

In his paper The Chemical Basis of Morphogenesis [60], Alan Turing proposed the morphogenetic mechanism called diffusion-driven instability, now known as the Turing mechanism. This is based on the simultaneous occurrence of two physical processes: reaction and diffusion of substances (morphogens) interacting in a very non-intuitive way. While Turing's approach was simple, this does not means he ignored other important aspects in morphogenesis. Turing wrote: "...it is proposed to give attention rather to cases where the mechanical aspect can be ignored and the chemical aspect is the most significant." His mechanism can be described briefly: A homogeneous steady state of the concentrations is stable to perturbations when diffusion is not present, but becomes unstable when the diffusion is introduced. Once this mechanism is triggered, the chemical concentrations of the reactants evolve in time away from homogeneity, typically forming discernible steady spatial structures. These are the so called Turing patterns. The Turing mechanism is triggered whenever four conditions hold, and the domain is sufficiently large [36]. These conditions are expressed in terms of the coefficients of the linearization of the system at the spatially homogeneous steady state, as well as the diffusion coefficients.

The roles of domain size and geometry in this process are well documented in the literature $[25,39,42]$. These were called "domain effects" by the British embryologist Conrad Hal Waddington [65]. In fact, both growth and curvature of the domain are quite important in determining the structure of emerging patterns in development [40], particularly those due to diffusion-driven instability. In [24], the authors present an account of what has been done so far in understanding this diffusion-driven instability on growing domains. They review those approaches where the size of the domain was treated as a bifurcation parameter (equivalently, quasi-static domain growth), as well as those which derive necessary conditions for the occurrence of a Turing bifurcation under stringent conditions, such as slow and isotropic growth [32]. Finally, they relax these assumptions and construct complicated history-dependent conditions generalizing the classical Turing conditions to uniform growth of a one-dimensional domain. The key point in the analysis they mention (which is recalled in [5]) is the nonautonomous nature of the reaction-diffusion system on a growing domain. This puts the analysis in a quite important mathematical perspective where much of the work is under current investigation. For instance, the notion of "steady-state" in a non-autonomous system has many non-equivalent operationalizations (i.e. plausible mathematical formalisms). One must carefully consider the history of a non-autonomous dynamical system to account for instabilities in a meaningful way, and in general this is not tractable analytically.

Besides stationary patterns, other behaviors are possible in reaction-diffusion systems, such as periodic oscillations. One well known mechanism for the emergence of an isolated periodic solution (limit cycle) is a Hopf bifurcation. This occurs in a nonlinear two-dimensional 
autonomous ordinary differential equation system as one parameter is varied. When one incorporates constant diffusion terms into a system whose homogeneous part exhibits a Hopf bifurcation, it is possible to obtain spatial patterns which oscillate in time. Additionally, there exist spatio-temporal oscillatory patterns occurring within the parameter values where the linear local analysis predicts only steady nonhomogeneous Turing patterns without any Hopf bifurcation at all [29]. In [66], the authors examine the interaction between stationary Turing patterns and oscillatory wave modes for obtaining oscillatory Turing-like patterns. This instability is sometimes known as a wave instability. We also mention [26], where the authors demonstrate oscillatory creation, travelling, and destruction of patterns in reactionadvection-diffusion scenarios on a static sphere, with the advection and compactness of the domain implicated in the oscillatory nature of the patterns.

We now describe what we mean by Turing-Hopf bifurcation. This spatio-temporal instability is algebraically characterized in terms of the parameter values of the system as follows. The first condition for the Turing bifurcation is that the real part of the eigenvalues of the Jacobian matrix associated with the reactive part at the equilibrium be negative (this happens when the Jacobian's trace is negative and its determinant is positive). In other words, this requires that the spatially homogeneous system is stable at the equilibrium. In order to introduce the Turing-Hopf mechanism, this condition is replaced by the real part of these eigenvalues crossing the imaginary axis (the real part passing through zero) with a nonzero imaginary part; meanwhile the remaining three conditions for the emergence of Turing patterns are maintained. This indicates a Hopf bifurcation of the homogeneous kinetic system, as well as instability due to spatial perturbations due to the Turing instability. In order for both instabilities to occur at precisely the same point, in general two parameters must be varied, so this is a codimension-2 bifurcation.

A number of authors have studied the Turing-Hopf bifurcation on fixed domains. Their approach covers a wide range, from numerical simulations to mathematical modeling in different areas. Namely, this instability has been studied in continuous population dynamical models $[1,54,67]$, in discrete time models [50], and in chemical reactions [22, 46, 51]. Other literature deals with various theoretical aspects of this bifurcation $[11,48]$. In the vicinity of a Turing-Hopf bifurcation, the competition between unstable modes coming from the Turing bifurcation and those coming from the Hopf can result in complex spatio-temporal patterns in reaction-diffusion systems [10, 35, 57].

In [5], the authors derived the necessary conditions on the parameter values for the occurrence of Turing and Turing-Hopf bifurcations for the FitzHugh-Nagumo equations. They considered two scenarios: a fixed square and an isotropically slowly growing square. The specific growth function they used was exponential in time. The numerical simulations reported in the above paper showed the richness of the emerging patterns in both scenarios for parameters where Turing and Turing-Hopf bifurcations occurred. A remarkable spatiotemporal behavior in the Turing-Hopf case was also presented. This was characterized by the appearance of oscillatory patterns. These consisted of a series of pairs of spirals which rotate (inwards and outwards) and change their intensity as time increased. For more details the reader is referred to sections 2.6 and 3.6 of the above mentioned reference. 
The FitzHugh-Nagumo model, derived independently by [15, 16] and [38], is a twocomponent simplification of the famous Hodgkin-Huxley model [19], which itself was proposed following experiments with the giant squid axon. The Hodgkin-Huxley equations model changes in the voltage potential across a surface membrane due to the movement of potassium and sodium ions (plus a small leakage current of other ions). In general, the FitzHugh-Nagumo caricature is a prototypical model for excitable media throughout a range of tissues and in other settings [23]. While many papers have studied FitzHugh-Nagumo kinetics in the context of Turing instabilities and pattern formation theoretically, only a few have studied biologically-motivated pattern formation using these kinetics. Work on the slime mold Dictyostelium discoideum has used excitable kinetics motivated by FitzHughNagumo in order to capture wave-induced patterning due to cAMP signalling and cell aggregation $[12,53]$, though we note that in [12] cells and the cAMP substrate are coupled via chemotaxis, and both studies assume an immobile recovery variable. We also mention similar pattern formation in neurogenesis [3], where FitzHugh-Nagumo kinetics were used to explain physiologically-relevant aspects of emergent patterning.

In the present paper, which can be seen as an extension of [5], we analyze the FitzHughNagumo reaction-diffusion model with the aim of detecting oscillatory patterns by means of the Turing-Hopf bifurcation on non-planar geometries. We do this on both a torus and a sphere, to demonstrate the effects of different kinds of curved manifolds. In both cases we consider the analysis without growth, as well as when these domains grow slowly and isotropically, with an exponential growth function. The use of exponential and slow growth allows for a mathematically simple analysis of the instability conditions. We will also demonstrate spatio-temporal patterns which are not predicted from this linear analysis, and we suspect multistability (of the partial differential equations, rather than the kinetic part) is implicated in these. We note that on many domains, multistability of several non-uniform patterns is generic, especially in manifolds with dimension greater than one $[2,20,21]$

While it is known that growth and curvature influence patterning in reaction-diffusion systems, the precise impact these effects have on pattern selection is only understood in very simple cases, and for particular kinds of nonlinear reaction kinetics. Here we will be interested in growing domains with different kinds of curvature. Specifically, we will consider both a sphere and a torus, the latter being an exemplar of nontrivial curvature. Locally on the surface of the sphere, the Gaussian curvature is constant, and essentially the space is locally isotropic. Spheres are also canonical domains for many biological studies in development, tumour growth, etc $[6,28,63]$. On a torus, however, the Gaussian curvature on the outermost part of the torus is different from the innermost, leading to a local anisotropy due to nonuniform curvature. This mathematical difference between the two surfaces, among other things, should lead to different kinds of modes being excited due to Turing instabilities, as well as possible nonlinear interactions such as competition between modes. This is of biological relevance, as local anisotropy is implicated in a variety of symmetry-breaking mechanisms in biology beyond symmetric patterning which one would expect from a Turing instability [7, 45, 61]. We demonstrate differences in patterning behaviors between these two geometries, suggesting that even this simple example of non-constant curvature can 
substantially impact pattern selection and evolution. This suggests further work on more complex curved domains in realistic biological settings should be carried out, as such pattern selection due to geometry has not been fully characterized.

It is often the case that the effects of slow growth do not drastically change the instability region [32], and that the overall patterns observed are qualitatively similar, differing primarily in the positioning of pattern elements which has suggested growth as a mechanism for robustness [8]. However, we will show here that comparing quasi-static reaction diffusion processes to ones on growing domains can radically change the kind of patterning observed for the FitzHugh-Nagumo kinetics. This immediately implies an important hysteresis in the evolution of the fully nonlinear pattern on a slowly growing domain, which we have not seen reported in the literature. Such a history-dependent effect is in the same spirit as [24], but is not related to the linear stability analysis, as it is due to the evolution of fully nonlinear patterns due to growth. Biologically, this suggests that growth can not only provide robustness of Turing patterns, but that it can fundamentally change the kind of pattern observed. Such nonlinear pattern evolution may help ameliorate some of the difficulties in understanding Turing patterning mechanisms in complex developmental settings [30].

The paper is organized as follows: in section 2, we describe the reaction-diffusion FitzHughNagumo system defined on the torus and sphere, as well as recalling the stability analysis for both Turing and Turing-Hopf bifurcations. In section 3, we compare the linear analysis of these bifurcations, with full numerical simulations on both geometries, in the case of static and growing manifolds. Finally section 4 contains a discussion and a brief list of relevant open problems which we think are worth investigating.

\section{FitzHugh-Nagumo on the torus and the sphere}

Here we develop the model equations on these manifolds. We also determine how the equations on a growing manifold appear on a static reference domain, which is necessary to

make the model amenable to numerical simulation. Finally, we recall stability criteria to detect Turing and Turing-Hopf bifurcations.

\subsection{The model and a basic analysis}

After an appropriate rescaling, the general form of a reaction-diffusion system defined on a fixed two-dimensional domain with two reactants is

$$
\begin{aligned}
& u_{t}=\Delta u+f(u, v ; c), \\
& v_{t}=d \Delta v+g(u, v ; c),
\end{aligned}
$$

where $d=D_{2} / D_{1}, D_{1}$ and $D_{2}$ are the diffusion coefficients of the reactants whose concentration are $u$ and $v$ respectively, $c$ is a kinetic parameter and $\Delta$ is the two dimensional Laplacian operator. When we add the initial and the boundary conditions the mathematical problem is well posed, as long as $f$ and $g$ are sufficiently well-behaved (usually these functions contain dissipative nonlinearities sufficient for this purpose). If the two dimensional domain is a surface embedded in a three-dimensional space, then $\Delta$ represents the Laplace-Beltrami operator acting on this surface, which can be defined via local coordinates on the manifold. 
The kinetics of the FitzHugh-Nagumo model can be written as $f(u, v ; c)=c\left(u-\frac{u^{3}}{3}+v+I_{0}\right)$ and $g(u, v ; c)=-(u-a+b v) / c$. Hence the system we consider takes the form

$$
\begin{aligned}
& u_{t}=\Delta u+c\left(u-\frac{u^{3}}{3}+v+I_{0}\right), \\
& v_{t}=d \Delta v-\frac{u-a+b v}{c}
\end{aligned}
$$

where $a, b, c$ and $d$ are positive parameters and $I_{0}$, in the original physiological interpretation, is an applied current. We note that $u$ typically has the interpretation of a membrane potential or voltage, and $v$ is a recovery variable, characterizing a relaxation process. We note that in general $u$ will take positive and negative values, corresponding to differences in an electrical potential [23]. As in [3, 12, 53], we are using these kinetics to consider a general excitable medium, rather than directly modelling two interacting chemical species or a specific electrochemical process. For this reason we need not think of $u$ and $v$ as concentrations, and hence do not require them to remain positive, as they generally will not for most parameter values.

In order to incorporate growth and curvature of the domain into a reaction-diffusion model, Plaza et al [47] used first principles to derive a general mathematical framework which takes into account both factors. We refer to this paper for details. We denote by $X$ a surface embedded in $\mathbb{R}^{3}$ which is parameterized as

$$
X(\xi, \eta, t)=(x(\xi, \eta, t), y(\xi, \eta, t), z(\xi, \eta, t))
$$

and define $h_{1}=\left\|X_{\xi}\right\|$ and $h_{2}=\left\|X_{\eta}\right\|$, where $\|\cdot\|$ is the Euclidean norm, and the subscripts denote partial differentiation. By assuming that both processes, reaction and diffusion take place on the surface $X$, and using conservation of mass, the system (1) becomes

$$
\begin{aligned}
& u_{t}=\frac{1}{h_{1} h_{2}}\left[\left(\frac{h_{2}}{h_{1}} u_{\xi}\right)_{\xi}+\left(\frac{h_{1}}{h_{2}} u_{\eta}\right)_{\eta}\right]-\partial_{t}\left(\ln \left(h_{1} h_{2}\right)\right) u+f(u, v ; c), \\
& v_{t}=\frac{d}{h_{1} h_{2}}\left[\left(\frac{h_{2}}{h_{1}} v_{\xi}\right)_{\xi}+\left(\frac{h_{1}}{h_{2}} v_{\eta}\right)_{\eta}\right]-\partial_{t}\left(\ln \left(h_{1} h_{2}\right)\right) v+g(u, v ; c) .
\end{aligned}
$$

We consider two specific manifolds to demonstrate differences due to curvature and growth. First, let us consider the isotropically growing torus

$$
X(\xi, \eta, t)=\rho(t)\left[\begin{array}{c}
(R+r \cos \eta) \cos \xi \\
(R+r \cos \eta) \sin \xi \\
r \sin \eta
\end{array}\right]
$$

where $R>r>0, \xi, \eta \in[0,2 \pi)$, and $\rho(t)>0$ is the growth function. The major and minor radii, $R$ and $r$, determine the overall geometry of the torus, which can be seen in Figure 1a for the case of $r=R / 3$. The growth function appears multiplying the three entries of $X$, and so the surface grows in the same proportion in each direction, i.e. isotropically. 
In order to obtain the model for our specific isotropically growing surface, we calculate $h_{1}^{2}$ and $h_{2}^{2}$. These are

$$
h_{1}^{2}=\left\|X_{\xi}\right\|^{2}=(R+r \cos \eta)^{2} \rho^{2} \quad \text { and } \quad h_{2}^{2}=\left\|X_{\eta}\right\|^{2}=r^{2} \rho^{2},
$$

respectively. By substituting (4) and (5) into (3), this system becomes

$$
\begin{gathered}
u_{t}=\frac{1}{\rho^{2} r^{2}}\left[\frac{r^{2}}{(R+r \cos \eta)^{2}} u_{\xi \xi}+u_{\eta \eta}-\frac{r \sin \eta}{R+r \cos \eta} u_{\eta}\right]-\frac{2 \dot{\rho}}{\rho} u+f(u, v ; c), \\
v_{t}=\frac{d}{\rho^{2} r^{2}}\left[\frac{r^{2}}{(R+r \cos \eta)^{2}} v_{\xi \xi}+v_{\eta \eta}-\frac{r \sin \eta}{R+r \cos \eta} v_{\eta}\right]-\frac{2 \dot{\rho}}{\rho} v+g(u, v ; c) .
\end{gathered}
$$

Next we consider the isotropically growing sphere parameterized by

$$
X(\xi, \eta, t) \equiv \rho(t)\left[\begin{array}{c}
\sin \eta \cos \xi \\
\sin \eta \sin \xi \\
\cos \eta
\end{array}\right],
$$

where $\xi \in[0,2 \pi], \eta \in[0, \pi]$, (see figure $1 b$ ) and $\rho(t)$ is the growth function. We again calculate $h_{1}^{2}$ and $h_{2}^{2}$ to find

$$
h_{1}^{2}=\left\|X_{\xi}\right\|^{2}=\rho^{2} \sin ^{2} \eta, \quad h_{2}^{2}=\left\|X_{\eta}\right\|^{2}=\rho^{2}, \text { then it follows } \frac{h_{1}}{h_{2}}=\sin \eta .
$$

Thus, the reaction-diffusion system we are going to consider on the sphere is

$$
\begin{gathered}
u_{t}=\frac{1}{\rho^{2}}\left(u_{\eta \eta}+\frac{1}{\sin ^{2} \eta} u_{\xi \xi}+\frac{\cos \eta}{\sin \eta} u_{\eta}\right)-\frac{2 \dot{\rho}}{\rho} u+f(u, v), \\
v_{t}=\frac{d}{\rho^{2}}\left(v_{\eta \eta}+\frac{1}{\sin ^{2} \eta} v_{\xi \xi}+\frac{\cos \eta}{\sin \eta} v_{\eta}\right)-\frac{2 \dot{\rho}}{\rho} v+g(u, v) .
\end{gathered}
$$

For both manifolds we will take the explicit form of the growth function $\rho(t)=\exp (k t)$. Hence the dilution terms in (6) and (9) simplify as $\dot{\rho} / \rho=k$. By redefining the kinetics as

$$
\begin{aligned}
& \tilde{f}(u, v ; c)=f(u, v ; c)-\frac{2 \dot{\rho}}{\rho} u=c\left(u-\frac{u^{3}}{3}+v+I_{0}\right)-2 k u, \\
& \tilde{g}(u, v ; c)=g(u, v ; c)-\frac{2 \dot{\rho}}{\rho} v=-\frac{u-a+b v}{c}-2 k v,
\end{aligned}
$$

the calculations for obtaining the parameter values for having each type of bifurcation (Hopf, Turing and Turing-Hopf) in the systems (6) and (9) is easier. We will sketch the results about these bifurcations derived in [4] and [5] in the next few sections, but refer to these papers for further details. 


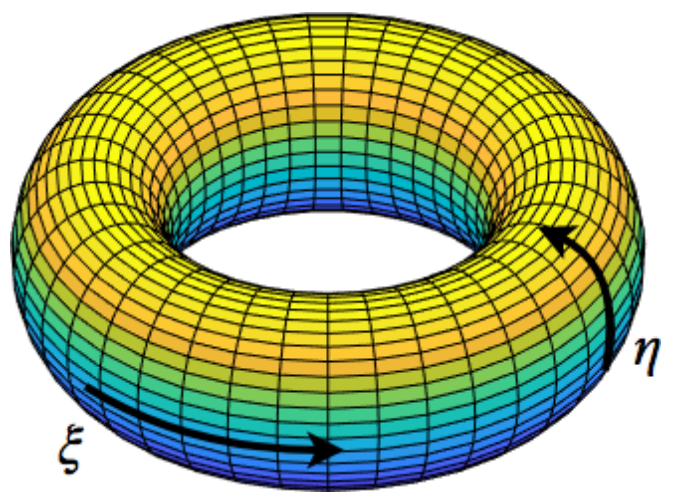

(a)

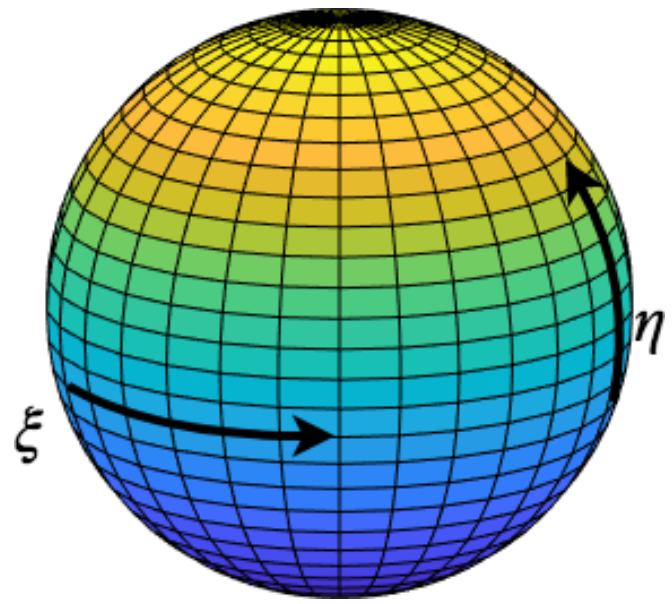

(b)

Figure 1: (a) Torus with parameterization given by the term enclosed in rectangular brackets in (4), (b) Sphere parameterized by the term enclosed in rectangular brackets in (7).

We note from [5] that by imposing the conditions

$$
\left(\frac{2 k}{c}-1+\frac{1}{b+2 k c}\right)>0 \quad \text { and } \quad\left(I_{0}+\frac{a}{b+2 k c}\right)>0
$$

we can guarantee that equations (10) have a unique positive real root and two complex conjugate roots. Hence, the homogeneous system associated with (6) and (9) has a unique real positive equilibrium which we denote $\left(u^{*}, v^{*}\right)$. This defines a unique steady and homogeneous solution for these systems, which is constant in time due to the use of exponential growth (e.g. the spatially homogeneous system is autonomous). In contrast, once we consider stability with regard to spatial diffusion, we have to consider the non-autonomous nature of the equations induced by growth, leading in some cases to time-dependent eigenvalues and more generally to the breakdown of exponential-in-time stability analysis $[17,18]$. Stability analysis for partial differential equations in this setting is only understood under relatively restrictive assumptions, which we now describe.

\subsection{Necessary conditions for Turing instability}

We are first interested in determining boundaries of the parameter space whereby a spatial perturbation to this homogeneous steady state will grow (e.g. the Turing space). In [32], the authors derived necessary conditions for diffusion-driven instability on a growing domain for a general growth function. This leads them to carry out the linear analysis around a time-dependent (homogeneous) reference solution of the corresponding reactiondiffusion system. In this case, the Turing parameter space has notable changes as time evolves. In [24], the authors expand on this idea and present evolving parameter spaces for the Schnakenberg and Gierer-Meinhardt models, where the history-dependence of the patterns is clearly demonstrated. In contrast to these studies, we consider slow exponential 
growth, for which the dilution rate becomes constant, and so our reference state is given by the homogeneous and stationary solution of our system given by the unique positive equilibrium point of the kinetics. The time-dependent reference state (Equation (11) in [32]) is equivalent to the spatially-homogeneous system for which we have a unique constant equilibrium. Homogeneous perturbations to the spatially homogeneous equilibrium will lead to a transient difference in the reference state, but these will decay exponentially quickly. If only spatial perturbations are added (e.g. with zero spatial mean), then the reference state is identically constant in time, and this is the case we will consider in our simulations.

Assuming that the growth is slow and isotropic, and after an appropriate time scaling, [32] derived the following necessary conditions for Turing bifurcation:

$$
\begin{aligned}
f_{u}+g_{v}-2 h_{*} & <0 \\
\left(f_{u} g_{v}-f_{v} g_{u}\right)-h_{*}\left(f_{u}+g_{v}\right)+h_{*}^{2} & >0 \\
\left(d f_{u}+g_{v}\right)-h_{*}(1+d) & >0 \\
{\left[\left(d f_{u}+g_{v}\right)-h_{*}(1+d)\right]^{2}-4 d\left[\left(f_{u} g_{v}-f_{v} g_{u}\right)-h_{*}\left(f_{u}+g_{v}\right)+h_{*}^{2}\right] } & >0
\end{aligned}
$$

where $h_{*}$ is related to the value of the dilution term and all the partial derivatives, $f_{(\cdot)}$ and $g_{(\cdot)}$, are evaluated at the unique positive equilibrium, whose existence is already guaranteed. As the growth in equations (6) and (9) is already isotropic and exponential, we can apply these conditions under the additional assumption that the growth rate is small (e.g. $k \ll 1)$. For exponential growth, the modification to the standard Turing conditions takes the form $h_{*}=2 k$. We then have the following conditions for instability of the spatially homogeneous steady state:

$$
\begin{array}{r}
{\left[c\left(1-u^{* 2}\right)-\frac{b}{c}-4 k\right]<0,} \\
{\left[1-\left(1-u^{* 2}\right)(b+2 k c)+\frac{2 b k}{c}+4 k^{2}\right]>0,} \\
\left\{\left(d c\left(1-u^{* 2}\right)-\frac{b}{c}\right)-2 k(1+d)\right\}>0, \\
{\left[d c\left(1-u^{* 2}\right)-\frac{b}{c}-2 k(1+d)\right]^{2}-4 d\left[1-\left(1-u^{* 2}\right)(b+2 k c)+\frac{2 b k}{c}+4 k^{2}\right]>0,}
\end{array}
$$

From the inequalities (16) and (18) the parameters $b, c, d$ and $k$ must satisfy the following inequalities

$$
c<c^{*} \quad \text { and } \quad d>\frac{b+2 k c}{c^{2}\left(1-u^{* 2}\right)-2 k c} .
$$

where $c^{*}$ is given below as the Hopf bifurcation boundary.

We note that for small growth rates $k$ necessary to derive the above conditions, the effect of growth is small $(O(k))$ compared to the standard Turing conditions for a static domain, so one could approximate the Turing space using such conditions. This approach is sometimes 
known as an adiabatic approximation. Additionally, the conditions above will not be valid for all time $t$, as the growth does not remain small indefinitely even for small $k$. However, as we

will show, it is sufficient to determine the instability boundaries for reasonable periods of time as long as $k$ is sufficiently small. We refer to [24] for a more detailed discussion of this point. We also remark that we will be interested primarily in the qualitative impacts on patterning below, rather than just the instability boundaries. We will show that in contrast to the instability conditions, even small and exponential growth can have a substantial impact on the evolution of patterns on manifolds.

\subsection{Conditions for a Hopf and Turing-Hopf bifurcations}

We recall from [5] the conditions for the emergence of a limit cycle of the homogeneous system associated with (2) by means of a Hopf bifurcation. For this purpose we choose the parameter $c$ as our bifurcation parameter. The critical value, $c^{*}$, of $c$ for which a Hopf bifurcation occurs in the spatially-homogeneous system is

$$
c^{*}=\frac{2 k+\sqrt{4 k^{2}+b-b u^{* 2}}}{1-u^{* 2}} .
$$

From here we have that $c^{*}$ is a real number if $\left(4 k^{2}+b-b u^{* 2}\right) \geq 0$. Moreover, it is defined whenever $u^{* 2} \neq 1$. Hence by using the Hopf bifurcation theorem [34], we conclude that for each $c>0$ such that $c>c^{*}$, the homogeneous system associated with the systems (6) and (9) has a limit cycle surrounding the positive equilibrium $\left(u^{*}, v^{*}\right)$. Additionally, it is easy to show that the emergent limit cycle is stable (e.g. the Hopf bifurcation is supercritical). Therefore, we have that for each $c>0$ such that $c>c^{*}$, the homogeneous version of the systems (6) and (9) has an attracting limit cycle surrounding the positive equilibrium.

Once we have the conditions for the occurrence of Turing and Hopf bifurcations, it is straightforward to state the corresponding conditions for the occurrence of the Turing-Hopf bifurcation. What we need is to keep the conditions (17)-(19) and impose the restriction $c>c^{*}$ on the bifurcation parameter $c$, which in turn means the condition (16), no longer holds. In this case, the sign of the real part of the eigenvalues of the Jacobian matrix at the equilibrium changes from negative to positive as the parameter $c$ increases beyond $c^{*}$.

Finally we note that in the absence of a spatial instability (e.g. growth of a nonzero spatial eigenmode), diffusion will stabilize any nonuniform perturbations, so that a pure Hopf bifurcation will lead to a spatially-homogeneous oscillation. However, once other spatial eigenmodes have positive growth rates, as in the Turing-Hopf bifurcation, this is no longer necessarily true, and the behavior of the system depends on competition between modes. Such analysis generally involves nonlinear amplitude (or Ginzburgh-Landau) equations involving the growth rates of eigenmodes [52]. We are not aware of any computations of such amplitude equations applicable to systems defined on curved manifolds, and these will be generically difficult to study even if one could derive them (as curvature destroys most ansatz solutions for Ginzburgh-Landau equations). Instead we will explore the system numerically to determine when spatial or temporal modes dominate, or when their competition leads to spatiotemporal behaviors. In addition to understanding when Turing or Hopf modes lead to 
different dynamics, we will also explore the impact of curvature and growth, and show how fully nonlinear patterns can be impacted by growth.

\section{Bifurcations and Numerical Simulations}

In this section we first compute diagrams given the conditions for Turing, Hopf, and Turing-Hopf bifurcations above in order to demarcate the parameter space based on the linear theory into regions where we expect different behaviors. We will then simulate equations (6) and (9) at various points in these parameter spaces to demonstrate solution behaviors on both the torus and the sphere. We note in particular that the linear theory will only give a rough indication of the solution behavior, as nonlinearity can dominate the solution structure for large times. Nevertheless, reaction-diffusion systems have been successfully studied using the linear theory, and so elucidating precisely when and how it succeeds and fails to predict patterning is exactly what Turing had in mind when he described using machines to explore his mechanism originally.

We use the commercially-available finite element solver COMSOL, version 5.3, with which we discretize the manifolds using second-order (quadratic) triangular finite elements on the surface of each manifold. In all reported simulations, we used a relative tolerance of $10^{-4}$, and fixed an initial time step of $10^{-6}$ (but let the solver increase the time step freely as the solution evolved). The default Backward Difference Formula solver was used, which uses adaptive variable-order implicit methods of order one (backward Euler) to five. This choice of finite element software allows for simple implementations of reaction-diffusion systems on manifolds. This is because the Laplace-Beltrami operator on a surface of dimension $n$ can be constructed from the Laplace operator in the ambient space $\mathbb{R}^{n+1}[13,14,43]$. In the case of the sphere, we discretize the manifold using 19,496 triangular elements, and for the torus we use 11,540 triangular elements.

Convergence of our COMSOL implementation in time was checked by restricting the maximum time step, and convergence in space was determined via using different numbers of finite elements, and comparing the norm of solutions over time and space. We also checked convergence of static domain simulations using the Matlab package Chebfun [58, 59], obtaining quantitatively identical simulations. Additionally, we compared these simulations to direct finite-difference solutions comparable to those obtained in [5], and simulations using the closest-point method [31], to ensure that the finite element scheme was resolving complex spatio-temporal behaviors.

Unless otherwise specified, we take initial data of the form $u(0, \eta, \xi)=u^{*}\left(1+W_{u}(\eta, \xi)\right)$ and $v(0, \eta, \xi)=v^{*}\left(1+W_{v}(\eta, \xi)\right)$, where $W_{u}$ and $W_{v}$ are normally distributed spatial Gaussian processes with zero mean and standard deviation $10^{-2}$. The form of these noise terms ensures that the noise will be typically very much smaller than the steady state value. These small spatial noise terms are sufficient to excite any unstable mode given by the Turing or Hopf bifurcations described previously. For a given geometry, we will use the exact same realization of the noise terms for each simulation, although we note that additional simulations were also used with different initial data to check the robustness of our results. For the sphere we take an initial radius $R_{0}$ which we vary across the simulations, and for the torus an 
initial major radius given by $R_{0}$ and minor radius given by $R_{0} / 3$. We run simulations for varying final times $T$, but always ensure that we are observing long-time behaviors rather than transients. In the growing cases these will typically correspond to a manifold which will grow exponentially up to 4 times its initial size. That is, $T=\ln (4) / k$ so that the final radius of the manifold is $R=\rho(T) R_{0}=4 R_{0}$. We compare static manifolds in the case of $k=0$ on both the smaller and the larger domains, in order to determine when growth matters; in other words we consider both $R=R_{0}$ and $R=4 R_{0}$ when $k=0$. Throughout this section we will display patterns on the torus and the sphere using a fixed size of the manifold for the static and growing cases, and clearly describe the actual size of the manifolds in the figure captions.

The detailed spatial and spatiotemporal dynamics we find are generally complex. However, we define three qualitatively distinct states: spatially uniform oscillations, spatially nonuniform stationary patterns, and spatiotemporal dynamics (sometimes referred to in the literature as chemical turbulence). The first of these is simply a spatially-extended oscillation of the homogeneous system undergoing an oscillation. The second are commonly referred to as Turing patterns. Finally the third behaviour is a complex interplay of spiral waves and pulses lacking any persistent recurrent patterning over long time scales [56].

\subsection{Turing, Hopf, and Turing-Hopf Bifurcations}

We now use the criteria outlined in sections 2.2-2.3 to deduce parameter ranges where we have Turing and Hopf bifurcations. Where the equilibrium state is unstable both due to a Hopf bifurcation and to a perturbation for a nonzero wavenumber, we also identify the Turing-Hopf region. We note, however, that such linear analysis will only give information about modes which grow for short time intervals, and that the long-time behavior can differ from these predictions. Near the codimension-2 point where the Hopf and Turing bifurcation boundaries intersect, we anticipate an interplay between spatial patterning and temporal oscillation, and so we will primarily focus near this region, for small growth rates $k$.

We show how these instability regions change as we vary the ratio of diffusions $d$, the bifurcation parameter $c$, and the growth rate $k$ in Figure 2. In the case of a static domain without growth $(k=0)$, we see that the Turing and Hopf instability boundaries merge at a single point, consistent with a codimension-2 Turing-Hopf bifurcation. For $0<k \leq 0.01$, the bifurcation structure is almost identical to the case without growth. However, for larger values of $k$, we see the pure Hopf bifurcation region shrink until it coincides with the boundary of the Turing space. We note, however, that the conditions derived are only valid for small values of $k$, and both nonlinear effects and history-dependent effects will occur as $k$ increases [24]. For this reason, we will primarily explore growth when $k$ is very small, in which case the bifurcation regions are almost identical to the static domain case.

We have explored several points in this parameter space numerically, in addition to other values of $a, b$, and $I_{0}$ which all have qualitatively similar parameter space diagrams. We indicate the type of patterns observed on the $k=0$ diagram in Figure 2, where the behaviour was observed in one of the simulations described in the next sections. These correspond to either spatially homogeneous oscillations, stationary spatial patterning, or spatio-temporal oscillations. We checked several simulations in the region where the spatially homogeneous 

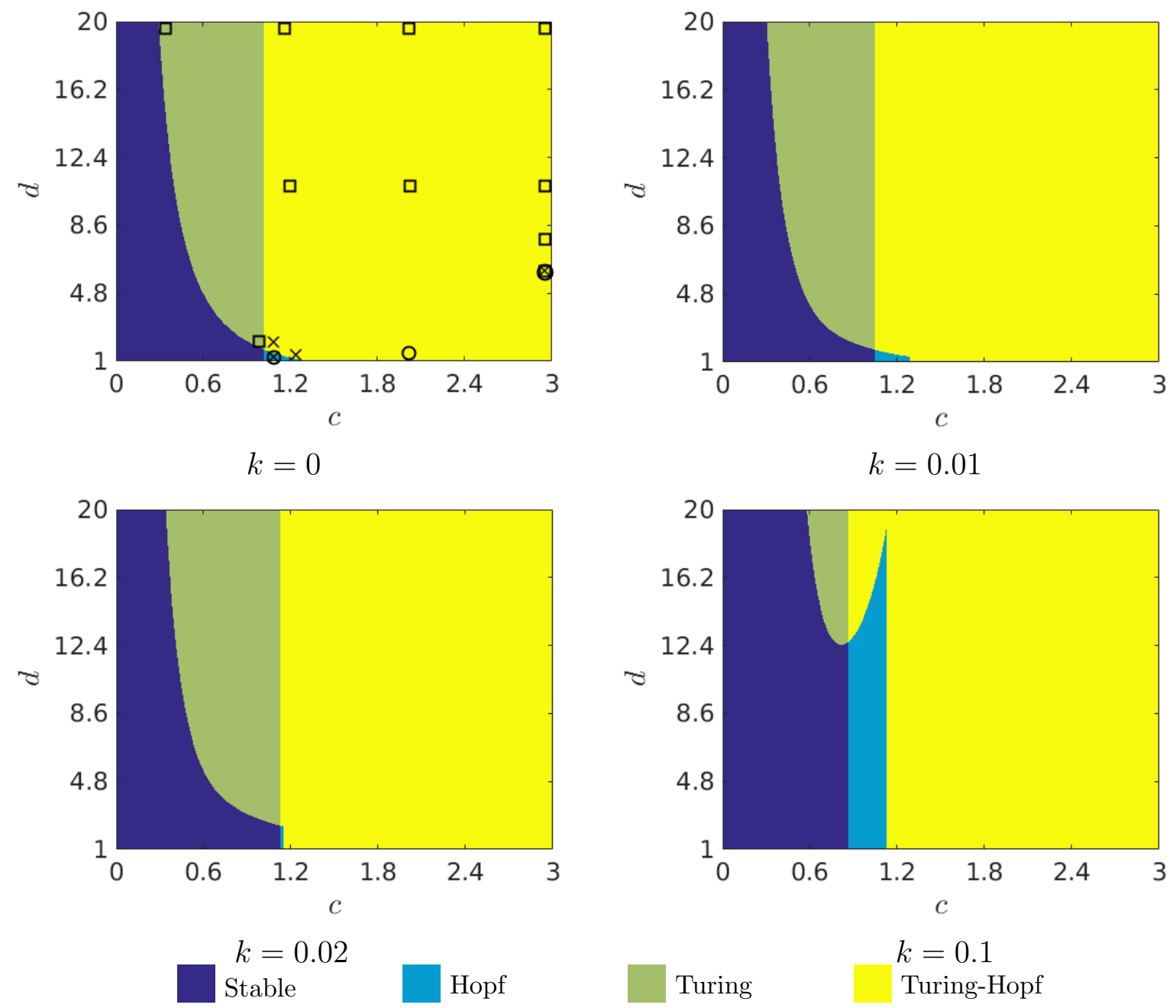

Figure 2: Parameter space diagrams illustrating regions where the unique spatially homogeneous steady state is stable, or unstable due to Turing or Hopf bifurcations. Here we used the parameters $a=0.6, b=0.99$, and $I_{0}=-0.6$ for varying values of $k$. For these parameters, the Hopf bifurcation boundary is characterized by $c^{*} \approx 1.0211$. For $k=0$ we mark the kind of behavors observed as spatially homogeneous oscillations by a $\circ$, stationary Turing patterns by a $\square$, and spatio-temporal oscillations by an $\times$. 
steady state was stable to confirm that it was stable for the initial data that we used for the remaining simulations.

We will only show a subset of these simulations that will be indicative of the kinds of dynamics observed throughout these parameter regimes. We remark that there are two interesting points where multiple solution behaviors were found, depending on the geometry (these are denoted by overlapping symbols in the $k=0$ panel of Figure 2). One of these is where spatio-temporal oscillations were found within the Hopf bifurcation regime, and the other is far into the Turing-Hopf regime. Finally, while we have restricted most of our analysis to the case where $k=0$, simulations for the small value of $k=0.001$ have comparable outcomes for small times (and almost identical parameter space regions), although differences do arise close to the boundaries of these regions, or for very large times when the growth is no longer sufficiently slow. We will therefore focus on the interior of these regions, and for short enough times such that the linear analysis is still valid. We note that the qualitative patterns we will present are apparent independent of the medium, but refer to the online version of the manuscript for colored figures. The colored scale used is an intense blue for the lowest value of $u$, and an intense red for the highest value of $u$.

\subsection{Patterning on the Torus}

We first consider the case of the reaction-diffusion system on the torus, given by (6). We ran 10 simulations of these reaction-diffusion systems using different realizations of the noisy initial conditions throughout the parameter space shown in Figure 2 (specifically where the symbols appear). While quantitative differences appeared between different initial data, such as different emergent patterns, in all cases the same qualitative behavior was observed. Here we will use a few choice examples to demonstrate the different kinds of patterning behavior emergent from this system.

In Figure 3 we consider simulations in the Turing space of Figure 2 for $k=0$ on a small torus (top row) and a large torus (middle row), and compare these with a growing torus (bottom row). In the static cases we observe spot patterns forming, which stabilize over the simulation time period. As expected, the larger torus admits many more spots, and the occasional spot which has curved due to neighboring interactions. The growing torus, however, admits a markedly different behavior, with spots growing and bending as the domain itself grows. That growth leads to different patterning is evident in comparing the final simulation times for the static large torus, and the grown torus, with the former admitting primarily a uniform spot pattern, and the latter showing a winding labyrinthine pattern coming from the initial spot formation before growth.

In Figure 4 we consider the same three tori using different parameters, yet still within the Turing space of Figure 2 for $k=0$. For these parameters, the static domains form twisted labyrinthine patterns across the torus. As one might expect, the larger torus has much finer patterning, with an occasional spot or defect in the labyrinthine structure. The growing case shows more regularity of the final patterned state by comparison to the larger static torus, but is otherwise qualitatively similar in structure.

Next we consider simulations within the Hopf regime, where the homogeneous steady state is not Turing unstable, but is Hopf unstable. We take $d=1$, implying the same 

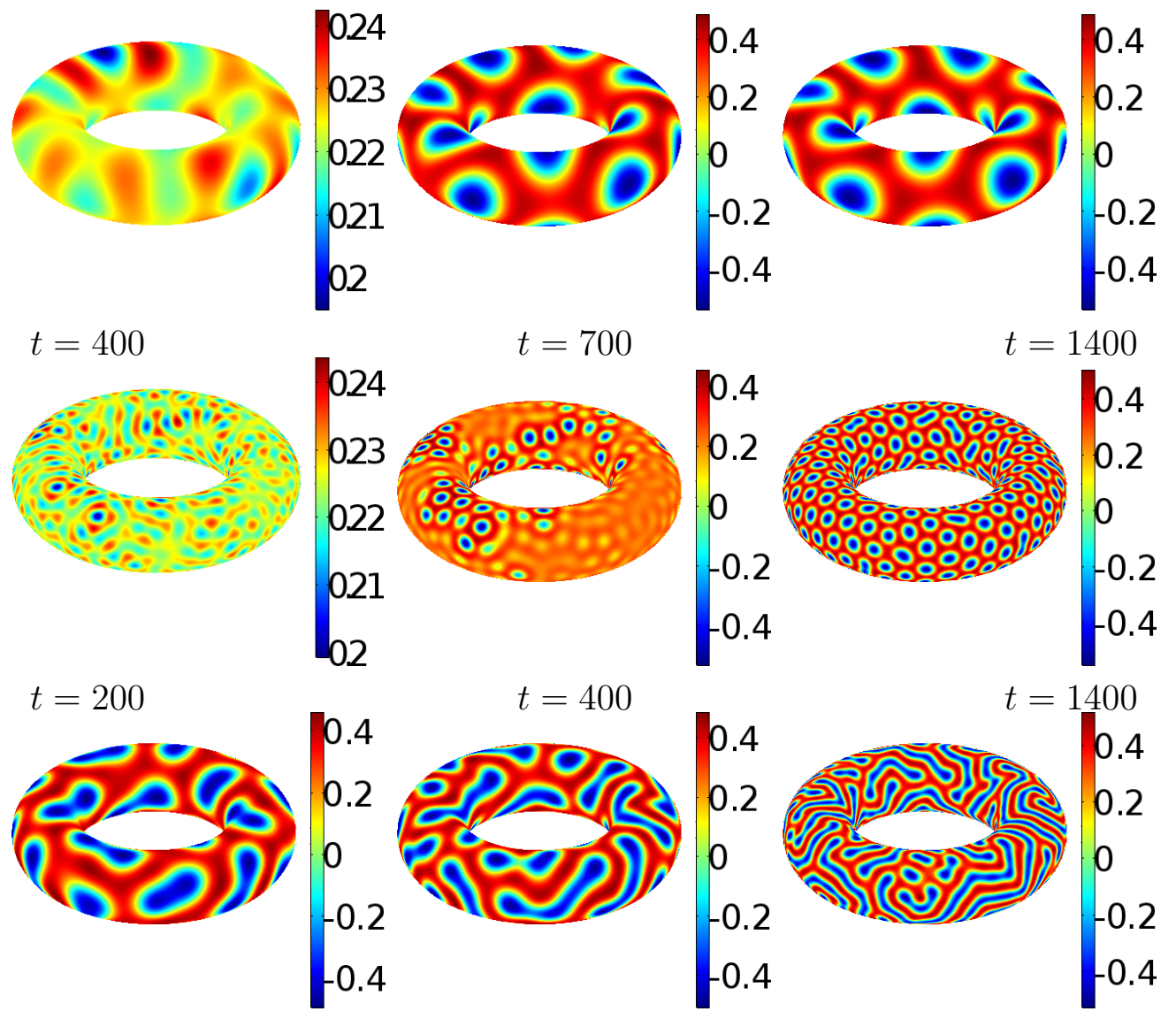

$t=400$

$t=700$

$t=1400$

Figure 3: Values of $u$ from solutions of equations (6). Here we used the parameters $a=0.6, b=0.99, c=1$, $d=1.75$ and $I_{0}=-0.6$ for two values of $k$. The first row uses $k=0, R_{0}=20$, the second row uses $k=0$, $R_{0}=80$, and the third row uses $k=0.001, R_{0}=20$. We note that in the growing case (third row), these three times correspond to when the manifold is $1.5,2$, and 4 times its initial size. 

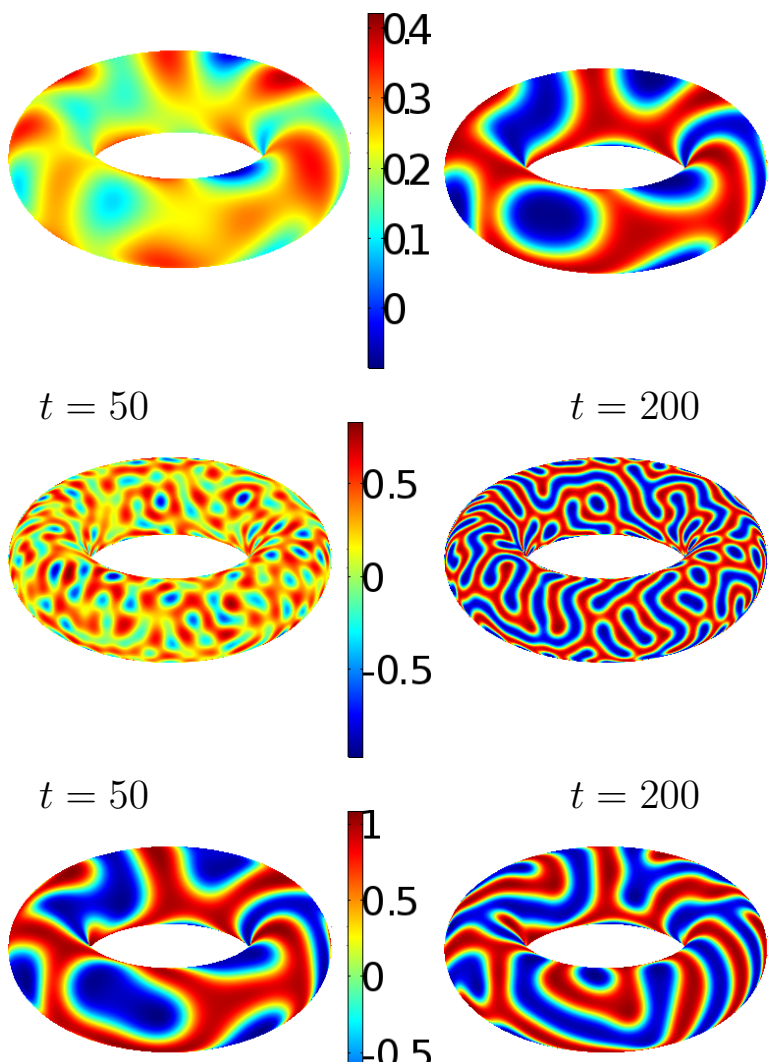

$t=200$
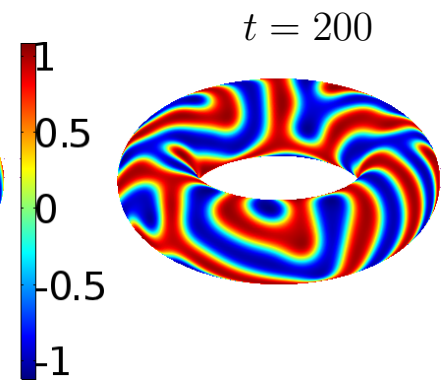
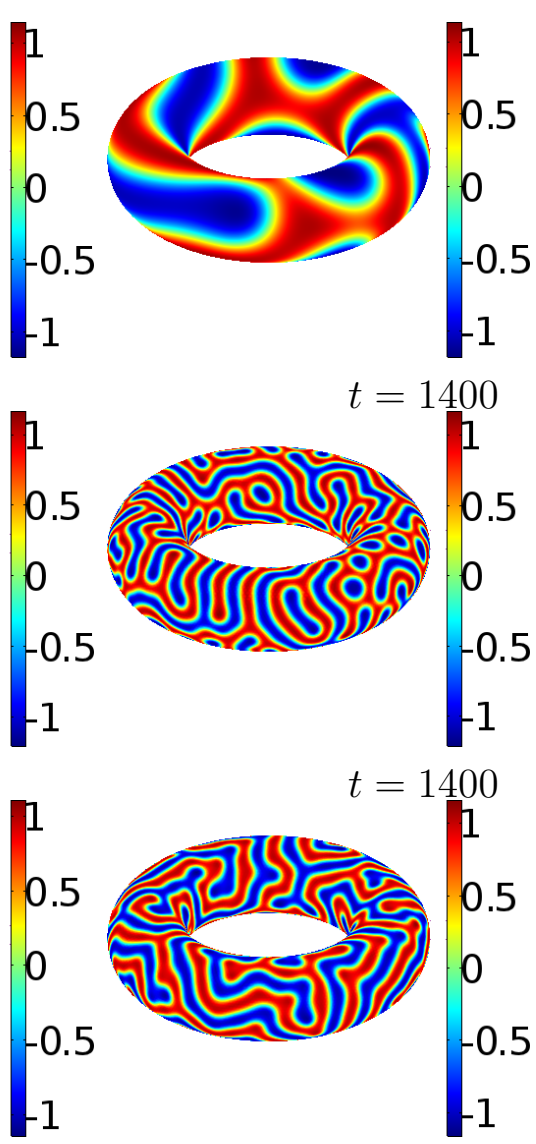

$$
t=700
$$

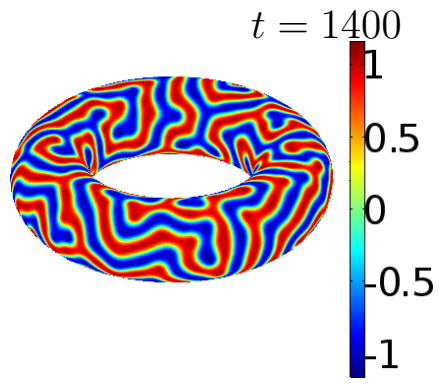

$t=1400$

Figure 4: Values of $u$ from solutions of equations (6). Here we used the parameters $a=0.6, b=0.99, c=0.5$, $d=20$ and $I_{0}=-0.6$ for two values of $k$. The first row uses $k=0, R_{0}=20$, the second row uses $k=0$, $R_{0}=80$, and the third row uses $k=0.001, R_{0}=20$. We note that in the growing case (third row), these three times correspond to when the manifold is $1.2,2$, and 4 times its initial size. 
diffusion rate between activator and inhibitor, which should not give rise to any spatial patterning via a Turing instability. Surprisingly, what we observe is a rapid oscillation which gives rise to spatial variation travelling around the torus in Figure 5, where again we consider two stationary tori and a growing one. We note that the spatial structure on the larger torus is much finer with more detail, consistent with what one expects on a larger domain, and that the growing torus then admits behavior on a variety of scales as it grows. Finally, we remark that these spatio-temporal behaviors fluctuate rapidly, with maximal and minimal spatial values changing quickly in time, and the spatial structure evolves via spiral waves and other complex patterns.

This surprising result could be due to subcriticality of the nearby Turing instability, which was recently discussed in [27]. This kind of bifurcation gives rise to multistability of homogeneous and inhomogeneous solutions, and our simulations indicate a different spatiotemporal solution attracts solution, rather than the homogeneous oscillation predicted by the Hopf bifurcation. The authors in [49] have also shown another mechanism by which Hopf bifurcations can excite Turing modes, even when the diffusion coefficients are equal, as they are in this case.

Solutions in Figure 2 sufficiently close to the intersection of the Hopf and Turing boundaries all exhibited qualitatively similar dynamics to those shown in Figure 5, including those only in the Hopf regime $\left(c>c^{*}\right)$ which were sufficiently close to this point. These spatiotemporal dynamics are then characteristic of not only what occurs in the Hopf regime, but also nearby in the Turing-Hopf regions of parameter space. For values of $c$ that are farther from this point, if the diffusion was sufficiently small then only spatially homogeneous oscillations were observed. Similarly, for large values of $d$, we observed primarily stationary Turing patterns forming, even when $c \geq c^{*}$. Specifically, we find only stationary patterns for $d \geq 7$ and $c \leq 3$, as shown by the squares in the $k=0$ case of Figure 2 . However, as these are very nonlinear effects, determining the boundaries of these behaviors is not presently a tractable problem.

Additionally, there are some parameter regions where either initial data or the size of the torus influenced the qualitative dynamics. In Figure 6 we plot solutions for $c=3$ and $d=5$, and see that the small torus and the growing torus both exhibit labyrinthine solutions comparable to Figure 4. However, for the larger torus we see Turing and Hopf modes directly competing, with local regions of striped patterns and spatio-temporal oscillations. Over time these oscillations destroy the patterned state and the final behaviour is a simpler travelling-wave like oscillation moving from one side of the torus to the other rapidly. This behaviour was observed across several realizations of the initial data, but for much larger initial data (e.g. when the Gaussian noise had standard deviations that were $O(1)$ ), we occasionally observed stationary labyrinthine patterning. Hence, whether the final behavior is a stationary labyrinthine pattern, or a simple spatio-temporal oscillation, depends on both the geometry and the initial data. For these parameters we never observed complex spatiotemporal dynamics which were not transient, suggesting that either Hopf or Turing modes would dominate the long-time behavior. 

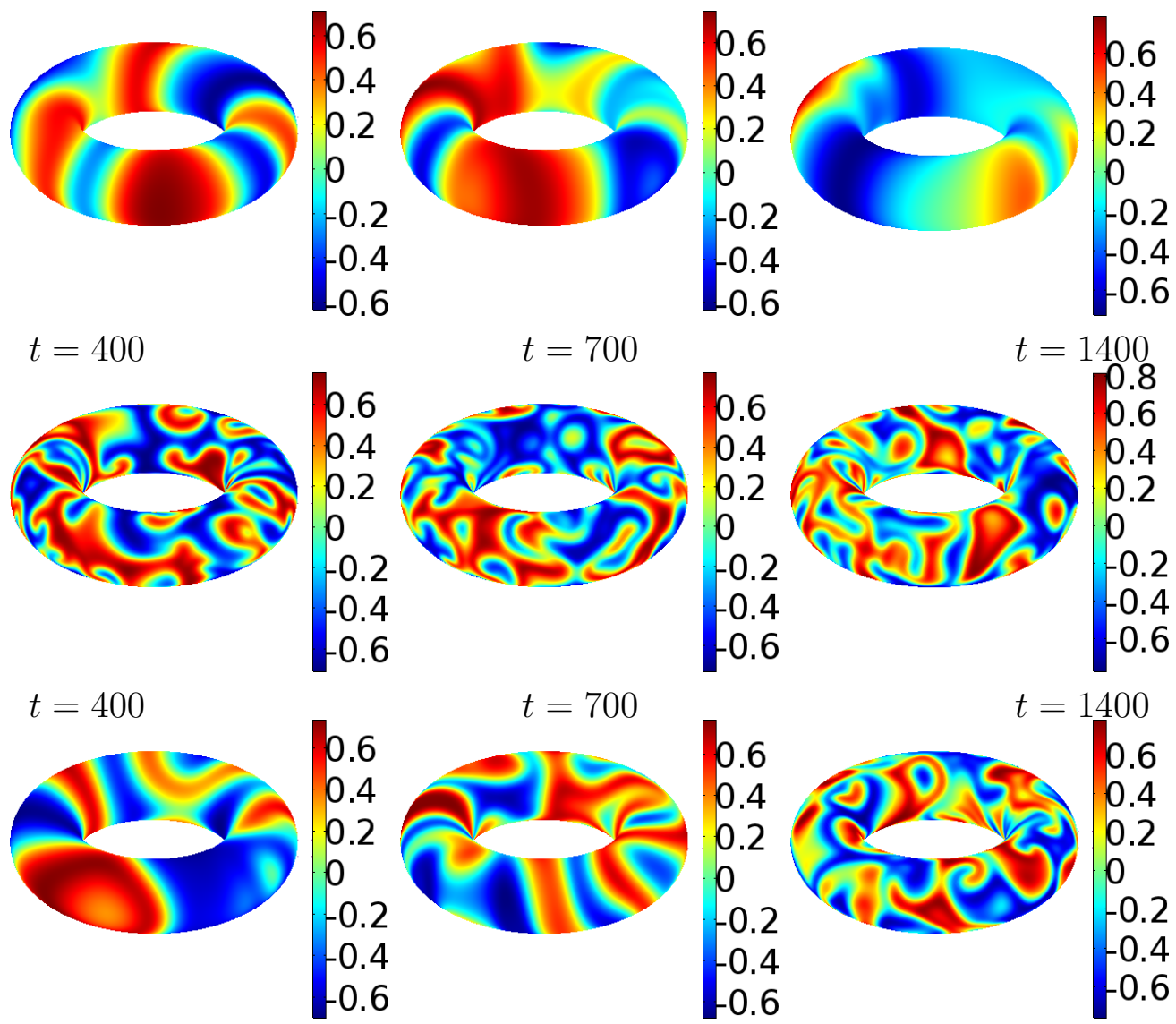

$t=400$

$t=700$

$$
t=1400
$$

Figure 5: Values of $u$ from solutions of equations (6). Here we used the parameters $a=0.6, b=0.99, c=1.1$, $d=1$ and $I_{0}=-0.6$ for two values of $k$. The first row uses $k=0, R_{0}=20$, the second row uses $k=0$, $R_{0}=80$, and the third row uses $k=0.001, R_{0}=20$. We note that in the growing case (third row), these three times correspond to when the manifold is $1.5,2$, and 4 times its initial size. 

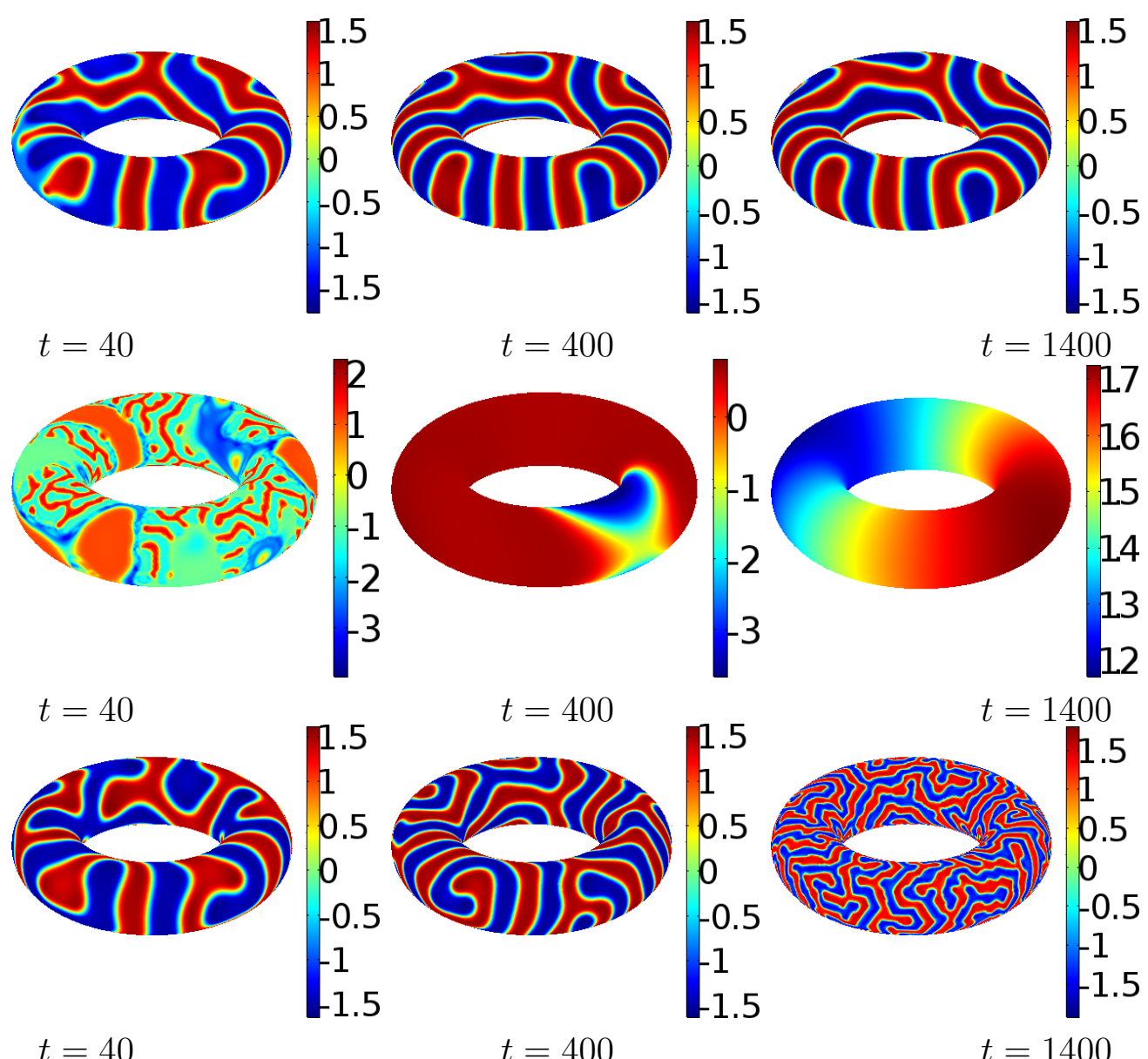

$$
t=40 \quad t=400 \quad t=1400
$$

Figure 6: Values of $u$ from solutions of equations (6). Here we used the parameters $a=0.6, b=0.99, c=3$, $d=5$ and $I_{0}=-0.6$ for two values of $k$. The first row uses $k=0, R_{0}=20$, the second row uses $k=0$, $R_{0}=80$, and the third row uses $k=0.001, R_{0}=20$. 


\subsection{Patterning on the Sphere}

We now consider simulations on a sphere. The topological differences between a torus and a sphere give rise to different symmetries, especially under the forms of isotropic growth used, so we want to consider geometries that are reasonably similar. One approach would be to set the radius of the sphere to be equal to the major radius of the torus. Alternatively, as we are patterning on the surface, we could choose sizes of the sphere that give comparable surface areas. The tori we have considered have surface areas of $4 \pi^{2} R^{2} / 3$, with $R$ the major radius. We note that this is $1 / 3$ the surface area of a sphere with a comparable outer radius, so we could scale the radius of the sphere by $1 / \sqrt{3}$ of the outer radius of the torus to achieve a surface of identical area. We simulated both choices, and will mention differences between them where they arise. For consistency of notation we will treat $R_{0}$ as the radius of the sphere.

We first demonstrate comparable simulations to Figures 3-4 on the sphere in Figures 7-8. These are again three different scenarios (a small sphere, a large sphere, and a growing sphere) in the case of parameters which give rise to spots in Figure 7, and parameters that lead to labyrinthine patterns in Figure 8. As in the toroidal case, we observe the influence of growth in both parameter regimes, and broadly see the same dynamics as on the torus despite differences in the manifold. We do note that in the cases of growing spots and stripes, locally on the surface of the manifold, the deformation of the spots is influenced via an isotropic scaling of the local curvature, whilst the torus has different scalar (e.g. Ricci or Gaussian) curvature inside as opposed to outside the torus. This difference in curvature undoubtedly influences the final patterned state, but remarkably no obvious effect is visually apparent, and observing the two we might claim they are, at least qualitatively, identical. For instance, considering the final pattern on the large static torus and sphere (the middle row and third column in both Figures 3 and 7), we note that, besides some defects, many of the spots are arranged with a sevenfold symmetry, despite difference in the local curvature. We do remark that the growing case, for both spots and stripes, does appear to 'conserve' the number of regions of high and low activator $u$, and so the growing case contains fewer connected regions than the static large sphere.

Analogously to Figure 5, we considered simulations of equations (9) in the pure Hopf regime, where the linear theory only anticipates spatially homogeneous oscillations about the steady state. In Figure 9 we see that the small and large static spheres, as well as the growing sphere, all admit spatio-temporal solutions. We observe simpler spatial structures on the small sphere, consisting of large waves travelling across the surface, in comparison to more complex local interactions between wavefronts in the larger sphere. In this case, the growing sphere admits solutions which appear to become finer in spatial structure as time goes on, suggesting that in this case, a quasi-static approximation of the domain would appropriately capture the dynamics of slow growth with respect to the size of the observed patterns.

We demonstrate an example of a detailed spatio-temporal behavior in Figure 10, using the same parameters as in Figure 9 on a small and a very large static sphere. We can see that the small sphere exhibits a large spiral-like region, which the solution is periodically 

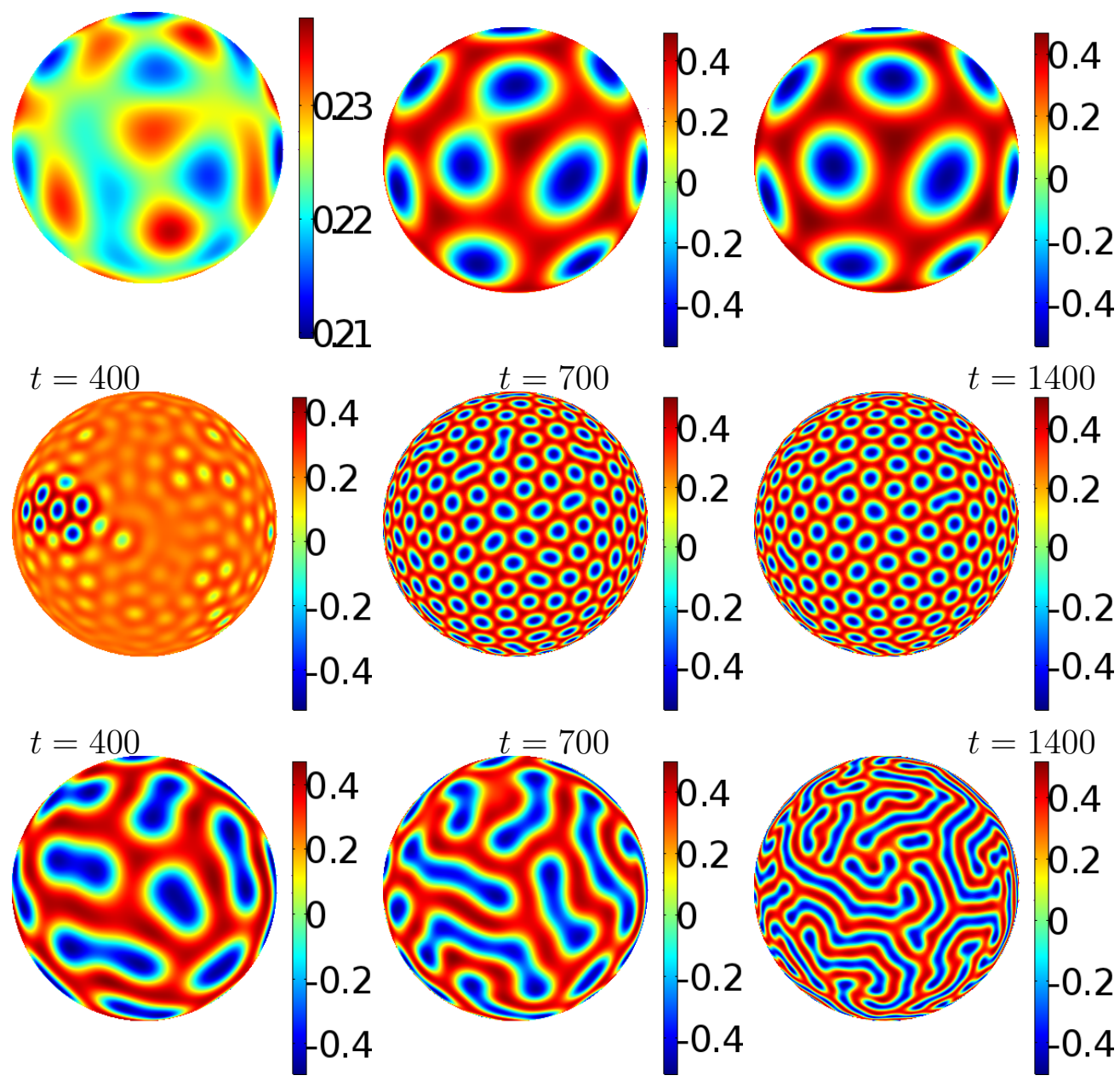

$t=400$

$$
t=700
$$

$$
t=1400
$$

Figure 7: Values of $u$ from solutions of equations (9). Here we used the parameters $a=0.6, b=0.99, c=1$, $d=1.75$ and $I_{0}=-0.6$ for two values of $k$. The first row uses $k=0, R_{0}=20$, the second row uses $k=0$, $R_{0}=80$, and the third row uses $k=0.001, R_{0}=20$. We note that in the growing case (third row), these three times correspond to when the manifold is $1.5,2$, and 4 times its initial size. 

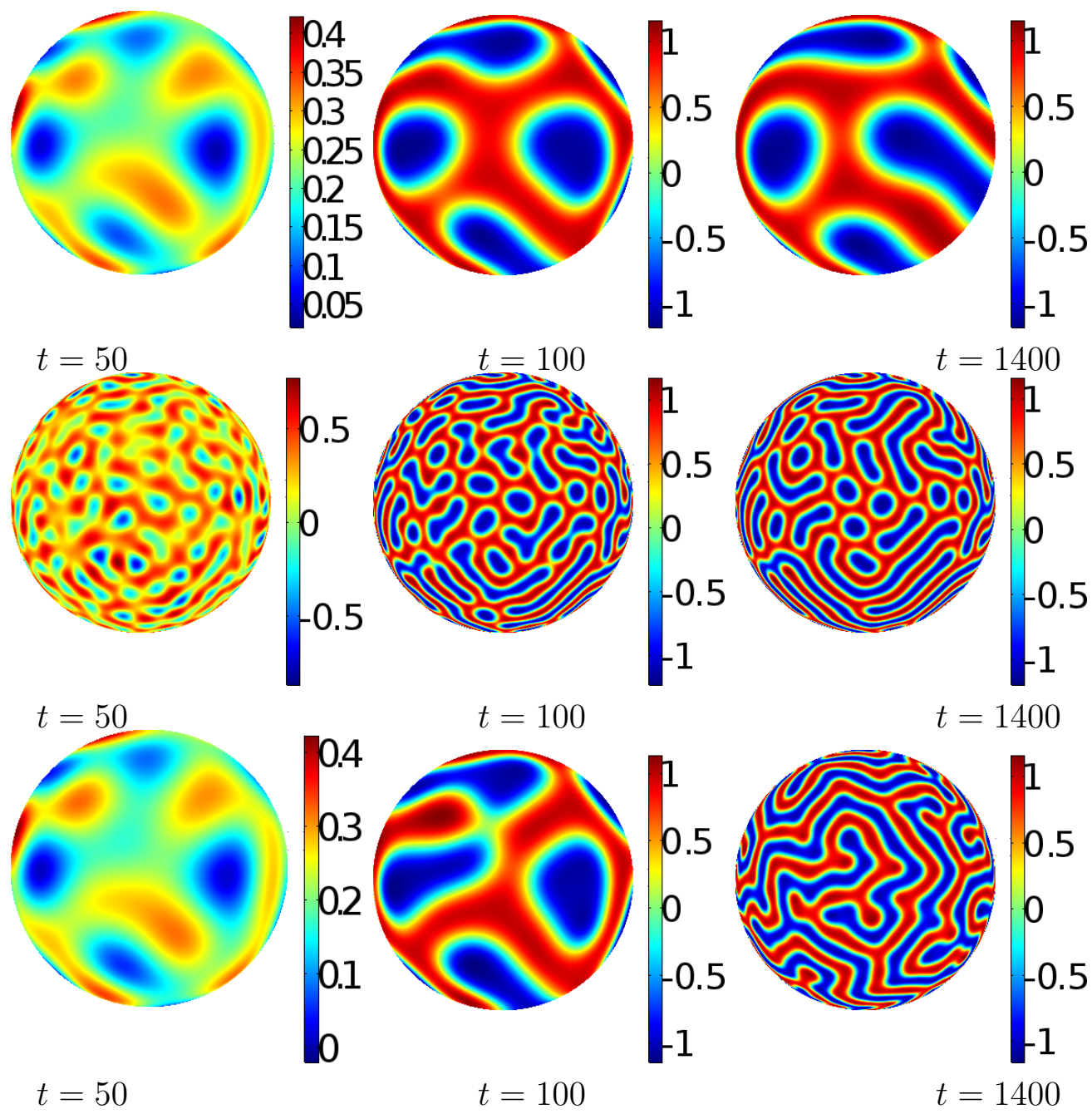

Figure 8: Values of $u$ from solutions of equations (9). Here we used the parameters $a=0.6, b=0.99, c=0.5$, $d=20$ and $I_{0}=-0.6$ for two values of $k$. The first row uses $k=0, R_{0}=20$, the second row uses $k=0$, $R_{0}=80$, and the third row uses $k=0.001, R_{0}=20$. We note that in the growing case (third row), these three times correspond to when the manifold is $1.2,2$, and 4 times its initial size. 

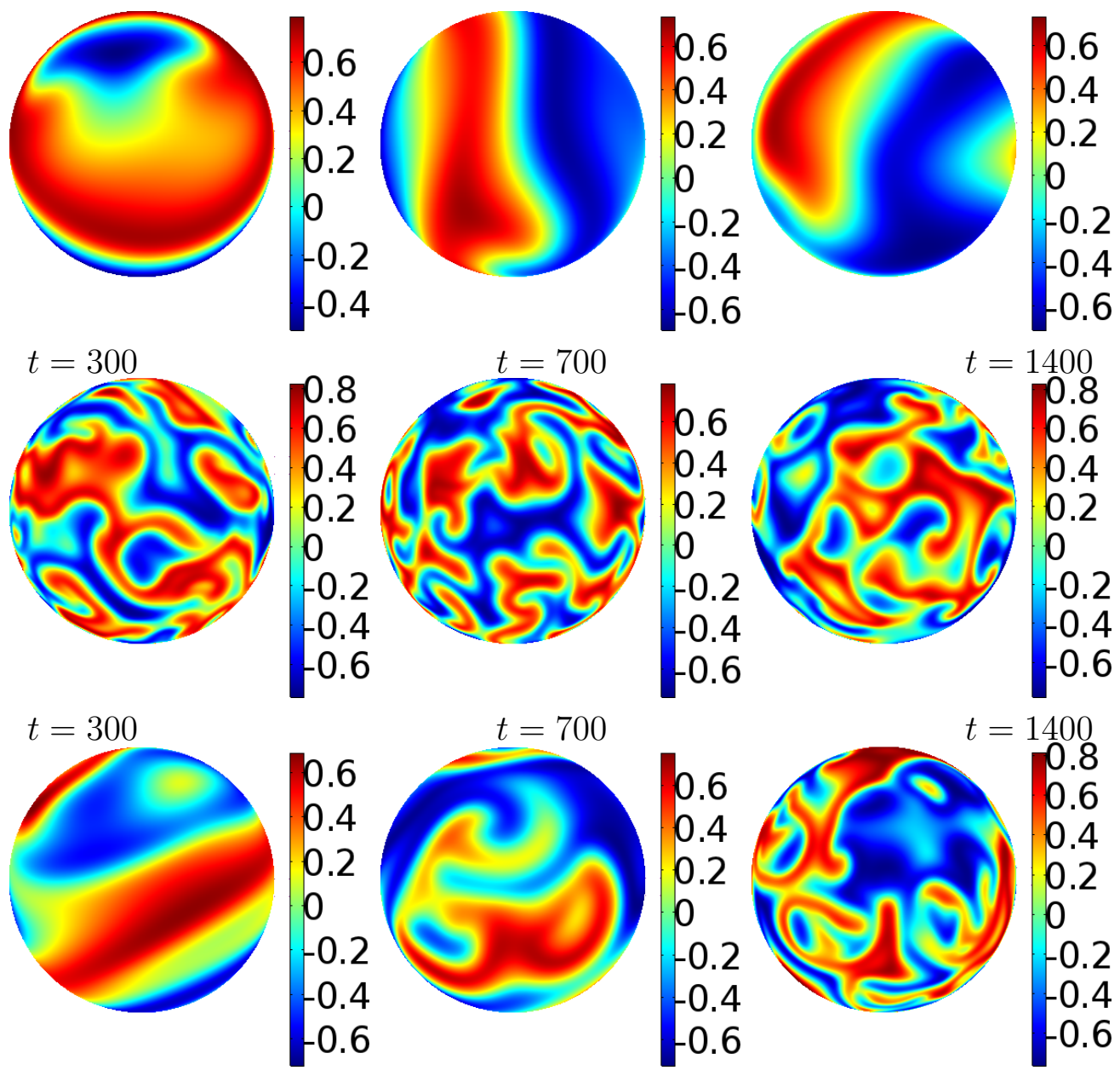

$t=300$

$t=700$

$t=1400$

Figure 9: Values of $u$ from solutions of equations (9). Here we used the parameters $a=0.6, b=0.99, c=1.1$, $d=1$, and $I_{0}=-0.6$. The first row uses $k=0, R_{0}=20$, the second row uses $k=0, R_{0}=80$, and the third row uses $k=0.001, R_{0}=20$.

emanating from. The large sphere demonstrates many interacting waves and oscillations all over its surface, giving rise to a complex picture with small spirals, loops, and pulsating spots. We note that this dependence on the size of the domain is characteristic of Turing patterns, as there are many more unstable wavemodes for a larger domain, but that here we have taken $d=1$ and so cannot, strictly speaking, be in the Turing regime.

We remark that in all of the above simulations, we have used $R_{0}$ to denote the radius of the sphere, and we have used comparable radii to those used for the major radius of the torus in the previous section. As mentioned at the beginning of this section, we also used a sphere of radius of $R_{0} / \sqrt{3}$ to achieve a surface with identical surface area to that of the torus simulations. In almost all cases, we observed qualitatively identical results, with only small quantitative differences due to a smaller manifold. In the case of the pure Hopf bifurcation (Figure 9) an important difference happens for the smaller spheres of comparable surface 

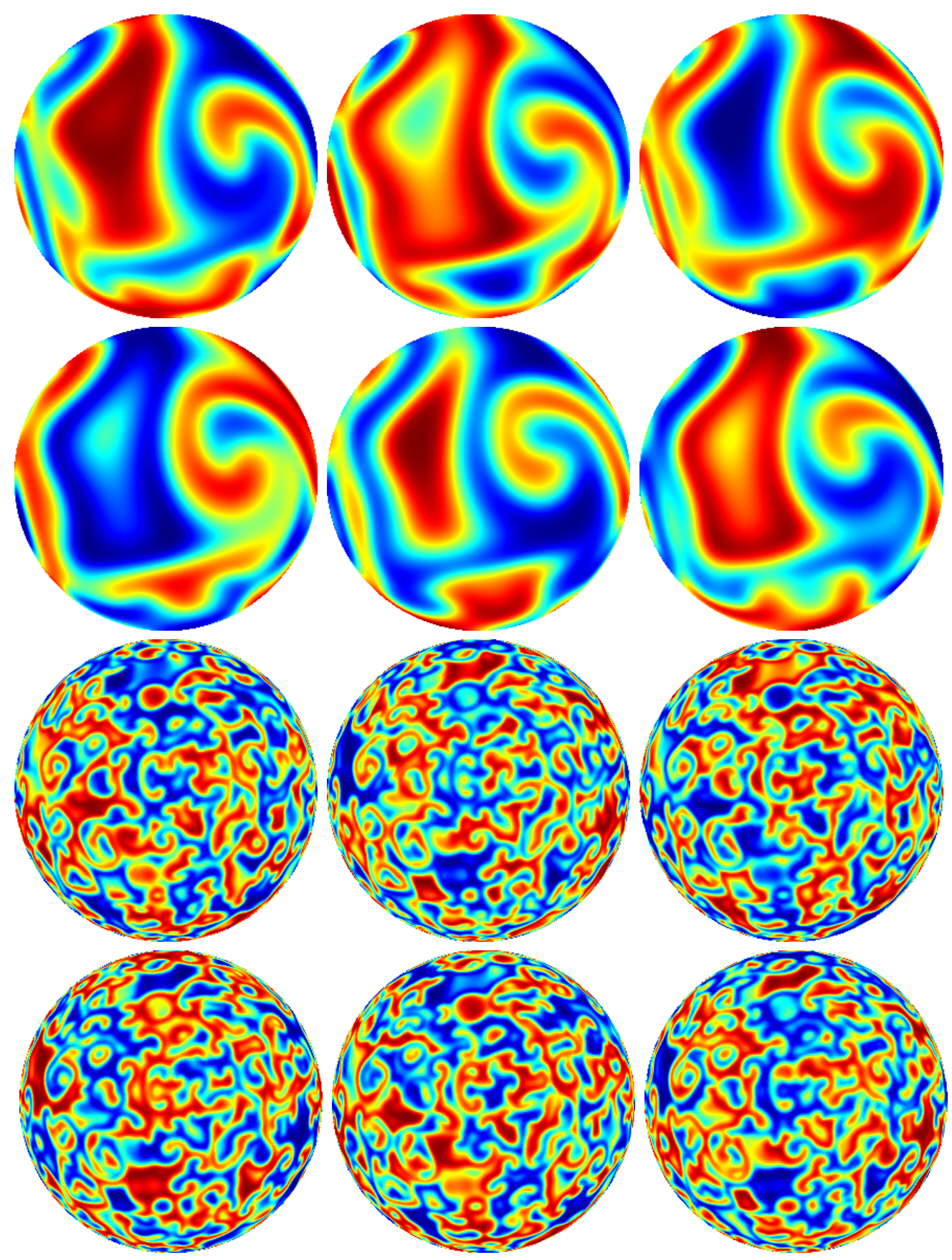

Figure 10: Values of $u$ from solutions of equations (9). Here we used the parameters $a=0.6, b=0.99$, $c=1.1, d=1 k=0, I_{0}=-0.6, R_{0}=40$ in the top two rows and $R_{0}=200$ in the bottom two rows. These are at times $t=900,904,908,912,916$, and 920 along each pair of rows. The colors are as in Figure 9 , with the minimum value of $u \approx-0.7$ and maximum $u \approx 0.7$. 
area to the tori from Figure 5. In the case of the small sphere (radius $20 / \sqrt{3}$ ) and the growing sphere, an initial spatio-temporal oscillation is observed that travels back and forth across antipodal points of the sphere. However, relatively quickly the spatial component of this oscillation dies away and the long-time behavior is in fact a spatially homogeneous oscillation. For the larger sphere (radius $80 / \sqrt{3}$ ), however, spatio-temporal oscillations can exist for precisely these parameters, as in Figure 9 . This suggests that determining which modes (Turing or Hopf) dominate the long-time stability depends on both the geometry of the manifold, and the history (e.g. whether or not growth occurred), as the sphere which grew to be of comparable size to the large one admits only spatially homogeneous oscillations.

Finally, we also consider simulations on the spherical geometry with parameters in the Turing-Hopf regime. For parameter values close to the Turing-Hopf point, we observe spatiotemporal oscillations as in the case of the torus. Far from it, we see a variety of stationary patterns as well as homogeneous and spatio-temporal oscillations. However, we typically see simpler behavior on the sphere than in the case of the torus. For instance, using the same parameters as in Figure $6(d=5$ and $c=3)$, we observe regions of spatial patterns and regions which are oscillating, but over time the oscillating regions destroy the patterned regions, leaving behind a sphere which is oscillating homogeneously. In comparison, for $d=7$ and $c=3$, we observe only patterned states comparable in structure to Figure 8.

\section{Conclusions}

In this paper we have used the general framework developed in [47] to study the influence of growth and curvature on a reaction-diffusion system. We have used the detailed analysis carried out in [5] to determine the parameter conditions for the occurrence of Turing and Hopf bifurcations. We presented the results of our study on the emergence of patterns supported by a reaction-diffusion system using FitzHugh-Nagumo kinetics. This was conducted in two domains, a torus and a sphere, and considering both static and growing domains.

It is well documented in the scientific literature that growth and curvature are crucial for the selection of patterns in reaction-diffusion systems [25, 39, 42, 47]. Of course, these influences were observed in the specific system we studied. The main features of the emerging patterns are determined by whether or not they are stationary in time. To a first approximation, this can be determined based on whether or not a Hopf bifurcation occurs (e.g. whether $c<c^{*}$ or $c>c^{*}$ ). This provides a necessary criterion for spatio-temporal patterns to emerge, but it is not sufficient, as for larger values of the diffusion ratio $d$, we still observe stationary patterns far into the Hopf regime. See Figure 2 for a rough characterization of the parameter space coming from the linear theory. Additionally, well beyond the Hopf bifurcation, oscillatory modes can dominate leading to simple spatial oscillations where most of the emergent patterning has been destroyed; see the middle row of Figure 6 for an example of this. The qualitative structure of the emergent patterns depends not only on the model parameters, but also on the geometry as well.

We note some interesting differences between these dynamics on the torus and on the sphere. The stationary Turing patterns in each case exhibit qualitatively similar behavior, although it is clear that the patterned structures themselves differ due to differences in local 
curvature. Stripes or spots on the sphere are in some sense locally isotropic, as there are no distinguished directions on the surface of a sphere. In contrast, stripes in the torus seem to have a slight preference for aligning along the major radius of the torus, likely due to differences in the eigenfunctions that are excited via the Turing instability. However, this small quantitative difference is very minor, and stripe stability and orientation only have a profound dependence on the geometry near parameter boundaries (see Figure 2). This is in agreement with what was found on the torus and the ellipsoid for different kinetics in [39].

In the Hopf and Turing-Hopf cases we see more dramatic differences between patterning on these manifolds, especially as the size of the torus and the sphere vary. In particular, consider the first row of Figure 5, and the middle row of Figure 6, where we see spatiotemporal oscillations or waves travelling along the torus only in one direction, without much structure in the transverse direction. This is a size-dependent effect, where spatial modes in the transverse direction are either never excited, or are dominated by Hopf modes, so that the motion is almost entirely in waves around the torus. On both manifolds, we can find some parameter regimes where competition between Turing and Hopf effects induces complex spiral waves and pulsating regions on the surface. Additionally, we refer to the sizedependent mode selection reported at the end of the previous section, where a sufficiently small sphere gave rise to only spatially homogeneous oscillations. This spatial homogeneity persisted even if the sphere grew from a small sphere. This is in sharp contrast to a static larger sphere, which gave rise to spatio-temporal patterning. Additionally, this homogeneous oscillation was not observed on static and growing tori with the same surface area.

Finally, we also discuss the nontrivial results for pure Turing patterns in static and growing domain cases shown in Figures 3 and 7. As the growth is slow, spots emerge on the smaller manifold before it grows very much, and so what we observe is the evolution of nonlinear stationary patterns into labrynthine-like arrangements which are fundamentally different to spot patterns observed on the small and large static domains which might adiabatically approximate the growing domain. This is in contrast to earlier studies of growth, which primarily observed robustness of patterning, but not qualitatively different patterns emerging from growth [8]. Additionally, this is a purely nonlinear phenomenon, and is unrelated to the stability analysis or mode selection effects which are more well understood, and which we also record here. This result especially calls for further work on the influence of growth in terms of pattern selection in fully nonlinear regimes, where patterns are already well developed before growth begins to change them.

There are many interesting open problems that could be pursued extending our work, and more generally the field of reaction-diffusion systems on growing manifolds. An important point to address is the history-dependence in the stability conditions, which becomes crucial for non-exponential growth, or over longer time scales. The authors in [24] outline a general procedure to do this, but restrict their attention to one-dimensional growth, remarking that linear and isotropic growth follows from their results. It is unclear how to proceed in the more general case. In particular, curvature and non-autonomous dynamics can both play a role due to the time-dependent Laplace-Beltrami operator corresponding to a growing manifold.

One could also investigate non-uniform domain growth in higher dimensions, as originally 
studied in [9] for a one-dimensional domain. Additionally, the investigation of anisotropic growth could also prove invaluable, as isotropic (or apical) growth is unable to recapitulate the complete range of complex biological structures observed in developing organisms [7, 45, 61]. Extending the stability analysis to this setting would involve a careful consideration of the kind of growth. In particular, it is not trivial to extend the results in $[24,32]$ to incorporate anisotropy or non-uniformity in the growth, which leads explicitly to spatial heterogeneity in the governing equations. We remark that not only does this complicate the bifurcation analysis, but a general modelling framework extending [47] to account for general non-uniform growth and anisotropy has not been presented in the literature.

Finally, we make a closing comment regarding the classification of the different schools of thought in explaining the origins of the biological forms and their mathematical formalizations. In [62] the author divides researchers into three schools of thought depending on the meaning given to the terms "patterning," "pattern formation" and "morphogenesis." These are the molecular, chemical, and mechanical views respectively. Alternatively, we propose that there are two schools of thinking about biological forms. One is an explanation given by evolutionary theory in terms of genetic modification due to natural selection. This viewpoint describes biological form following a teleology according to past selection pressures. We are not satisfied with this kind of historical account of form; while useful, it does not give a complete view into the development of the current biological structures. Quoting Wolpert [64]: "DNA provides the program that controls the embryo development and leads it along the epigenesis." Once again, everything in the embryo, including the forms, lies in the DNA. This is in stark contrast to a growing body of experiment which questions the gene-centric view of evolution [41].

The other school of thinking, called structuralism traces back at least to Goethe's times. Its finished form was due to the great Scottish scholar, D'Arcy Wentworth Thompson. His views on the underlying mechanisms, not just of the biological forms but for every single form in Nature, were written in an elegant prose and plenty of erudition in his grand opus $O n$ Growth and Form [55]. There, in the case of living organisms, Thompson does not suggest that heritage is unimportant in the forms organisms already have, but in addition to heritage, there exist physical constraints acting on every portion of matter across scales in time and space. The genes are not acting alone. They are part of matter which itself obeys physical laws. The matter is under physical structural constraints which have different origins ranging from those physical, chemical, mechanical, etc. Some of these can be formulated in terms of mathematical equations.

In these terms, we recognize ourselves on the side of the structuralist school of thought. While the model we have explored here is electrochemical in nature, we believe that the incorporation of growth is an important step toward a more thorough characterization of the physical and chemical basis of morphogenesis. This is in line with the mechanochemical models described in [33, 37, 44], where there is an important feedback between the physical constraints imposed by mechanics, and chemical patterning mediated via reaction and diffusion. Such constraints can include important geometric considerations, and it is here that we think our results help connect classical reaction-diffusion systems with such mechanochemical 
models. As discussed in the introduction, this is in line with Turing's approach to consider first the simplest setting whereby symmetry-breaking can be observed, and then to consider such aspects which had been first neglected [60].

Acknowledgements. FSG is grateful for the hospitality of the Wolfson Centre for Mathematical Biology of the Mathematical Institute, University of Oxford during his three months academic visit, where some final parts of this research were carried out. He also acknowledges facilities provided by the Institute Mittag-Leffler at Djursholm, Stockholm during his two weeks stay in the Fall of 2018.

[1] M. Baurmann, T. Gross, U. Feudel, Instabilities in spatially extended predatorprey systems: Spatio-temporal patterns in the neighborhood of Turing-Hopf bifurcations, J. Theor. Biol, 245, pp. 220-229, 2007.

[2] P. Borckmans, G. Dewel, A. De Wit, D. Walgraef, Turing bifurcations and pattern selection. In: Chemical waves and patterns, Springer, Dordrecht, pp. 323-363, 1995.

[3] J.H. Cartwright, Labyrinthine Turing Pattern Formation in the Cerebral Cortex, J. Theor. Biol., 217(1), pp.97-103, 2002.

[4] J.A. Castillo, F. Sánchez-Garduño, P. Padilla, Patrones de Turing-Hopf en dominios con crecimiento, Gaceta de la RSME, 18(2), pp. 269-286, 2015.

[5] J.A. Castillo, F. Sánchez-Garduño, P. Padilla, A Turing-Hopf bifurcation scenario for pattern formation on growing domains, Bull. Math. Biol., 78(7) pp. 1410-49, 2016.

[6] M.A. Chaplain, M. Ganesh, I.G. Graham, Spatio-temporal pattern formation on spherical surfaces: numerical simulation and application to solid tumour growth, J. Math. Biol., 42(5), 387-423, 2001.

[7] F. Corson, O. Hamant, S. Bohn, J. Traas, A. Boudaoud, Y. Couder, Turning a plant tissue into a living cell froth through isotropic growth. Proc. Natl. Acad. Sci., 106(21):8453-8458, 2009.

[8] E.J. Crampin, E.A. Gaffney, P.K. Maini, Reaction and diffusion on growing domains: Scenarios for robust pattern formation, Bull. Math. Biol., 61, pp. 1093$1120,1999$.

[9] E. Crampin, W. Hackborn, P.K. Maini, Pattern formation in reaction-diffusion models with nonuniform domain growth. Bull. Math. Biol., 64(4):747-769, 2002.

[10] A. De Wit, G. Dewel, P. Borckmans, Chaotic Turing-Hopf mixed mode, Phys. Rev. E 48, R4191-R4194, 1993. 
[11] R. Dilão, Turing Instabilities and Patterns Near a Hopf Bifurcation, Appl. Maths. Comput. 164(2) pp. 391-414, 2005.

[12] D. Dormann, B. Vasiev, C.J. Weijer, Propagating waves control Dictyostelium discoideum morphogenesis. Biophysical chemistry, 72(1-2), pp. 21-35, 1998.

[13] G. Dziuk, C.M. Elliott, Finite elements on evolving surfaces. IMA J. Num. Anal. 27(2):262-292, 2007.

[14] G. Dziuk, C.M. Elliott, Finite element methods for surface pdes. Acta Numerica 22:289-396, 2013.

[15] R. FitzHugh, Mathematical models of threshold phenomena in the nerve membrane. Bull. Math. Biol., 17(4):257-278, 1955.

[16] R. FitzHugh, Impulses and physiological states in theoretical models of nerve membrane. Biophys. J. 1(6):445-466, 1961.

[17] J. Gjorgjieva and J. Jacobsen, Turing Patterns on Growing Spheres: The Exponential Case, Disc. Cont. Dyn. Sys., Supplement pp. 436-445, 2007.

[18] J.K. Hale, A. P. Stokes, Conditions for the stability of nonautonomous differential equations, J. Math. Anal. Appl., 3(1):50-69, 1961.

[19] A.L. Hodgkin, A.F. Huxley, A quantitative description of membrane current and its application to conduction and excitation in nerve. J. Physiol. 117(4):500-544, 1952.

[20] A. Hunding, Dissipative structures in reaction-diffusion systems: Numerical determination of bifurcations in the sphere. J. Chem. Phys. 72(9):5241-5248, 1980.

[21] O. Jensen, V.O. Pannbacker, G. Dewel, P. Borckmans, Subcritical transitions to turing structures. Phys. Lett. A 179(2):91-96, 1993.

[22] W. Just, M. Bose, S. Bose, H. Engel, E. Schöll Spatiotemporal dynamics near a supercritical Turing-Hopf bifurcation in a two-dimensional reaction-diffusion system, Phys. Rev. E 64, pp. 026219-1 026219-12, 2001.

[23] J.P. Keener, J. Sneyd, Mathematical physiology, vol. 1. Springer, New York, 1998.

[24] V. Klika, E. A. Gaffney, History dependence and the continuum approximation breakdown: the impact of domain growth in Turing's instability. Proc. R. Soc. A, 473: 20160744, 2017.

[25] F. Kneer, E. Schöll, M.A. Dahlem, Nucleation of reaction-diffusion waves on curved surfaces. New J. Phys. 16(5):053010, 2014. 
[26] A.L. Krause, A.M. Burton, N.T. Fadai, R.A. Van Gorder, Emergent structures in reaction-advection-diffusion systems on a sphere. Phys. Rev. E 97(4):042215, 2018 .

[27] M. Kuznetsov, A. Kolobov, A. Polezhaev, Pattern formation in a reaction-diffusion system of fitzhugh-nagumo type before the onset of subcritical turing bifurcation. Phys. Rev. E 95(5):052208, 2017.

[28] H. Levine, W.J. Rappel, Membrane-bound Turing patterns, Phys. Rev. E., 72(6), 061912, 2005.

[29] R-T. Liu, S-S Liaw and P.K. Maini, Oscillatory Turing Patterns in A Simple Reaction-Diffusion System. J. Kor. Phys. Soc., 50, No. 1, pp. 234-238, 2007.

[30] L. Marcon, J. Sharpe Turing patterns in development: what about the horse part? Curr. Opin. Genetics Dev., 22(6):578-584, 2012.

[31] C.B. Macdonald, B. Merriman, S.J. Ruuth, Simple computation of reactiondiffusion processes on point clouds. Proc. Natl. Acad. Sci., 110(23):9209-9214, 2013.

[32] A. Madzvamuse, E. A. Gaffney, P. K. Maini, Stability analysis of non-autonomous reaction-diffusion system: the effects of growing domains, J. Math. Biol. 61, pp. 133-164, 2010.

[33] P.K. Maini, Spatial and spatio-temporal patterns in a cell-haptotaxis model. J. Math. Biol. 27, 507-522, 1989.

[34] J.E. Marsden, M. McCracken, The Hopf bifurcation and its applications. SpringerVerlag, New York, 1976.

[35] M. Meixner, A. De Wit, S. Bose, H. Engel, E. Schöll, Generic spatiotemporal dynamics near codimension-two Turing-Hopf bifurcations, Phys. Rev. E, 55(6):6690, 1997.

[36] J.D. Murray, Mathematical Biology II: Spatial Models and Biomedical Applications, Springer Verlag, New York Inc., 2003.

[37] J.D. Murray, G.F. Oster, A.K. Harris, A mechanical model for mesenchymal morphogenesis. J. Math. Biol. 17, 125-129, 1983.

[38] J. Nagumo, S. Arimoto, S. Yoshizawa, An active pulse transmission line simulating nerve axon. Proc. IRE 50(10):2061-2070, 1962.

[39] S. Nampoothiri, A. Medhi Role of curvature and domain shape on turing patterns. arXiv preprint arXiv:170502119, 2017. 
[40] C.M. Nelson, R.P. Jean, J.L. Tan, W.F. Liu, N.J. Sniadecki, A.A. Spector, C.S. Chen, Emergent patterns of growth controlled by multicellular form and mechanics. Proc. Natl. Acad. Sci., 102(33):11594-11599, 2005.

[41] D. Noble, Physiology is rocking the foundations of evolutionary biology. Exp. Phys., 98(8), pp.1235-1243, 2013.

[42] M. Núñez-López, G. Chacón-Acosta, J. Santiago, Diffusion-driven instability on a curved surface: Spherical case revisited. Braz. J. Phys. 47(2):231-238, 2017.

[43] M.A. Olshanskii, X. A. Xu, trace finite element method for pdes on evolving surfaces. SIAM J. Sci. Comp., 39(4):A1301-A1319, 2017.

[44] G.F. Oster, J.D. Murray, A.K. Harris, Mechanical aspects of mesenchymal morphogenesis. J. Embryol. Exp. Morph. 78, 83-125, 1983.

[45] A. Peaucelle, R. Wightman, H. Höfte, The control of growth symmetry breaking in the arabidopsis hypocotyl. Curr. Biol., 25(13):1746-1752, 2015.

[46] J. E. Pearson, Complex Patterns in a Simple System. Science, New Series, 261(5118), pp. 189-192, 1993.

[47] R.G. Plaza, F. Sánchez-Garduño, P. Padilla, R.A. Barrio , P.K. Maini, The Effect of Growth and Curvature on Pattern Formation. J. Dyn. Diff. Equat. 16, No. 4, pp. 1093-1121, 2004.

[48] M.R. Ricard, On Turing-Hopf instabilities in Reaction-Diffusion systems. Biophy. Rev. Lett, 3, 1 \& 2, pp. 257-274, 2008.

[49] M. R. Ricard, S. Mischler. Turing instabilities at Hopf bifurcation. J. Nonlin. Sci. 19(5), pp. 467-496, 2009.

[50] L.A.D. Rodrigues, D. C. Mistro, S. Petrovskii, Pattern Formation, Long-Term Transients, and the Turing-Hopf Bifurcation in a Space- and Time-Discrete Predator-Prey System. Bull. Math. Biol. 73(8), pp. 1812-1840, 2011.

[51] A. Rovinsky, M. Menzinger, Interaction of Turing and Hopf bifurcations in chemical systems. Phys. Rev. A, 46 (10), pp. 6315-6322 1992.

[52] W. van Saarloos, M. van Hecke, P.C. Hohenberg. "Amplitude equations for pattern forming systems." Fundamental Problems in Statistical Mechanics 8:245-278, 1994.

[53] N.J. Savill, P. Hogeweg, Modelling morphogenesis: from single cells to crawling slugs. J. Theor. Biol. 184, pp. 229-235, 1996. 
[54] H.B. Shi, S. Ruan, Spatial, temporal and spatiotemporal patterns of diffusive predator-prey models with mutual interference. IMA J. Appl.Math, 80(5), 1534$1568,2015$.

[55] D.W. Thompson, On growth and form. Cambridge University Press, Cambridge, 1917.

[56] S.M. Tobias, E. Knobloch, Breakup of spiral waves into chemical turbulence, Phys. Rev. Lett., 80(21):4811, 1998

[57] C.M. Topaz, A. J. Catllá, Forced patterns near a Turing-Hopf bifurcation, Phys. Rev. E 81:026213, 2010.

[58] A.Townsend, L.N. Trefethen, An extension of chebfun to two dimensions. SIAM J Sci. Comp., 35(6):C495-C518, 2013.

[59] A. Townsend, H. Wilber, G.B. Wright, Computing with functions in spherical and polar geometries I. the sphere. SIAM J. Sci. Comp., 38(4):C403-C425, 2016.

[60] A.M. Turing, The Chemical Basis of Morphogenesis, Phil. Trans. Roy. Soc. Lond. Series B, Biol. Scs., Vol. 237, No. 641, pp. 37-72, 1952.

[61] S. Ubeda-Tomás, R. Swarup, J. Coates, K. Swarup, L. Laplaze, G.T. Beemster, P. Hedden, R. Bhalerao, M.J. Bennett, Root growth in arabidopsis requires gibberellin/della signalling in the endodermis. Nat. Cell Biol. 10(5):625, 2008.

[62] S. Urdy: On the evolution of morphogenetic models: machano-chemical interactions and an integrated view of cell differentiation, growth, pattern formation and morphogenesis. Biol. Rev., 87, pp. 786-803, 2012.

[63] C. Varea, J.L. Aragón, R.A. Barrio, Turing patterns on a sphere. Phys. Rev. E., 60(4), 4588, 1999.

[64] L. Wolpert, The triumph of the embryo, Courier Corporation; 2008.

[65] C.H. Waddington, Principles of Embryology, Allen and Unwin, London, 1956.

[66] L. Yang, I.R Epstein, Oscillatory Turing patterns in reaction-diffusion systems with two coupled layers. Phys. Rev. Lett. 20 No. 17, pp. 178303-1-178303-4, 2003.

[67] J.-F. Zhang, W.-T. Li B, X. P. Yan, Hopf bifurcation and Turing instability in spatial homogeneous and inhomogeneous predator-prey models. Appl. Math. Comput. 218, pp. 1883-1893, 2011. 